\title{
Condensins: universal organizers of chromosomes with diverse functions
}

\author{
Tatsuya Hirano ${ }^{1}$ \\ Chromosome Dynamics Laboratory, RIKEN Advanced Science Institute, Wako, Saitama 351-0198, Japan
}

Condensins are multisubunit protein complexes that play a fundamental role in the structural and functional organization of chromosomes in the three domains of life. Most eukaryotic species have two different types of condensin complexes, known as condensins I and II, that fulfill nonoverlapping functions and are subjected to differential regulation during mitosis and meiosis. Recent studies revealed that the two complexes contribute to a wide variety of interphase chromosome functions, such as gene regulation, recombination, and repair. Also emerging are their cell type- and tissue-specific functions and relevance to human disease. Biochemical and structural analyses of eukaryotic and bacterial condensins steadily uncover the mechanisms of action of this class of highly sophisticated molecular machines. Future studies on condensins will not only enhance our understanding of chromosome architecture and dynamics, but also help address a previously underappreciated yet profound set of questions in chromosome biology.

During the past two decades, we have witnessed genomics and epigenomics quickly entering the central stage in biology and immensely transforming our understanding of gene functions, cell differentiation, development, biodiversity, and evolution. There is no doubt that this trend will continue. To fully "decode" genomic and epigenomic information, however, understanding their three-dimensional organization and dynamics is essential. In this sense, elucidation of higher-order chromosome structure will remain at the frontier of modern biology. From a historical point of view, a main branch of chromosome research had focused on structural and biochemical dissection of "condensed" chromosomes that become visible during cell divisions, eventually leading to the discovery of a class of multisubunit protein complexes collectively referred to as condensins. The founding member of condensins (now known as condensin I) was identified from Xenopus egg extracts as a major component of chromosomes that plays a crucial role in assembling chromosomes in the cell-free extracts.

[Keywords: chromosome condensation; sister chromatid separation; condensins; SMC proteins]

${ }^{1}$ Correspondence

E-mail hiranot@riken.jp

Article is online at http://www.genesdev.org/cgi/doi/10.1101/gad.194746.112.
Concurrent genetic studies in different model organisms provided strong lines of evidence that condensin I is essential for proper condensation and segregation of chromosomes in vivo. Remarkably, even bacteria and archaea turn out to have condensin-like complexes, and many eukaryotic species have a second condensin complex (condensin II). Most recently, ample evidence has been accumulating that condensins' functions are not limited to chromosome condensation and segregation in mitosis (or meiosis): They participate in a wide range of chromosome functions supporting genome stability, cell differentiation, and development. Hence, condensins are now recognized as universal organizers of chromosomes conserved among the three domains of life. In this review, I summarize and discuss recent progress in the field of condensins. Emphasis is placed on the evolutionary landscape of the chromosome organizers and the differential yet coordinated actions of the two different condensin complexes in eukaryotes. For recent reviews on this topic, see also Hirano (2005), Hudson et al. (2009), and Wood et al. (2010).

\section{Evolutionary landscape}

\section{Eukaryota}

It is currently known that there exist (at least) two different types of condensin complexes, known as condensins I and II, among eukaryotes. The two complexes share the same pair of SMC2 and SMC4 subunits, both belonging to the structural maintenance of chromosomes (SMC) family of chromosomal ATPases (Hirano 2006). Each complex has a unique set of three non-SMC subunits (i.e., CAP-D2, CAP-G, and CAP-H for condensin I, and CAPD3, CAP-G2, and CAP-H2 for condensin II). CAP-D2 and CAP-D3 (and CAP-G and CAP-G2) are distantly related to each other and have a degenerated repeat motif called HEAT repeats (Neuwald and Hirano 2000). On the other hand, CAP-H and CAP-H2 are members of the kleisin family of SMC-interacting proteins (Schleiffer et al. 2003). Electron microscopic analyses have visualized the highly characteristic architecture of condensin I (Anderson et al. 2002), and protein-protein interaction assays using recombinant subunits have revealed the geometry of the subunits within each complex (Fig. 1A; Onn et al. 2007). 
Condensin I is conserved from yeast to humans (Table 1; Hirano et al. 1997; Sutani et al. 1999). When condensin II was first discovered from vertebrate cells (Ono et al. 2003; Yeong et al. 2003), none of the corresponding subunits specific to condensin II was found in fungi such as Saccharomyces cerevisiae and Schizosaccharomyces pombe. It was therefore hypothesized that condensin II might be a new invention in evolution that provided an additional level of organization and rigidity for organisms with large chromosomes (Ono et al. 2003). However, information from an ever-growing number of eukaryotic genomes that have been sequenced since then strongly suggests that the original view is wrong. In fact, both condensins I and II are almost ubiquitous in eukaryotes, and only a limited number of organisms have condensin I only (Fig. 1B). There is no apparent relationship between the possession of condensin II and the size of genomes among eukaryotic species. The implication is that the last common ancestor of eukaryotes possessed both condensins I and II and that some species have lost condensin II during evolution. Moreover, a phylogenetic tree indicates that loss of condensin II occurred independently multiple times in different kingdoms during evolution (Fig. 1B).

\section{Bacteria/archaea}

Most if not all species of bacteria and archaea have a single condensin-like complex composed of three subunits: SMC, ScpA, and ScpB (hereafter referred to as the SMC-ScpAB complex) (Graumann and Knust 2009). On the other hand, some species belonging to a subclass of $\gamma$-proteobacteria, which includes Escherichia coli, have a distinct complex composed of MukB, MukE, and MukF (referred to as the MukBEF complex) instead of SMC-ScpAB (Hiraga 2000). Increasing lines of evidence from structural and genetic studies suggest that the MukBEF complex shares some functional properties with SMC-ScpAB, although the similarities between the corresponding subunits (SMC) MukB, ScpA/MukF, and ScpB/MukE) are rather limited at the amino acid sequence level. The core of the bacterial condensin complex is a homodimer of SMC/MukB subunits, and ScpA/MukF is likely to function as a kleisin (Fennell-Fezzie et al. 2005). The subunit-subunit interac-
A

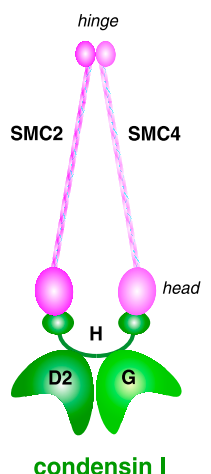

condensin I

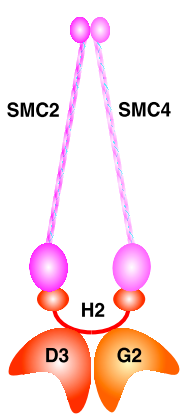

condensin II

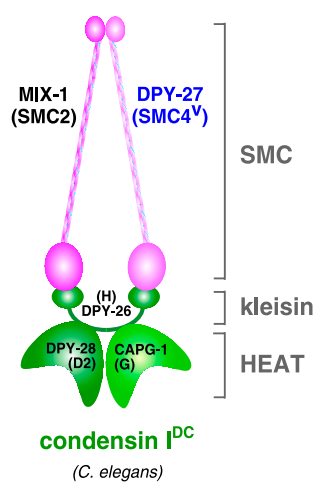

B

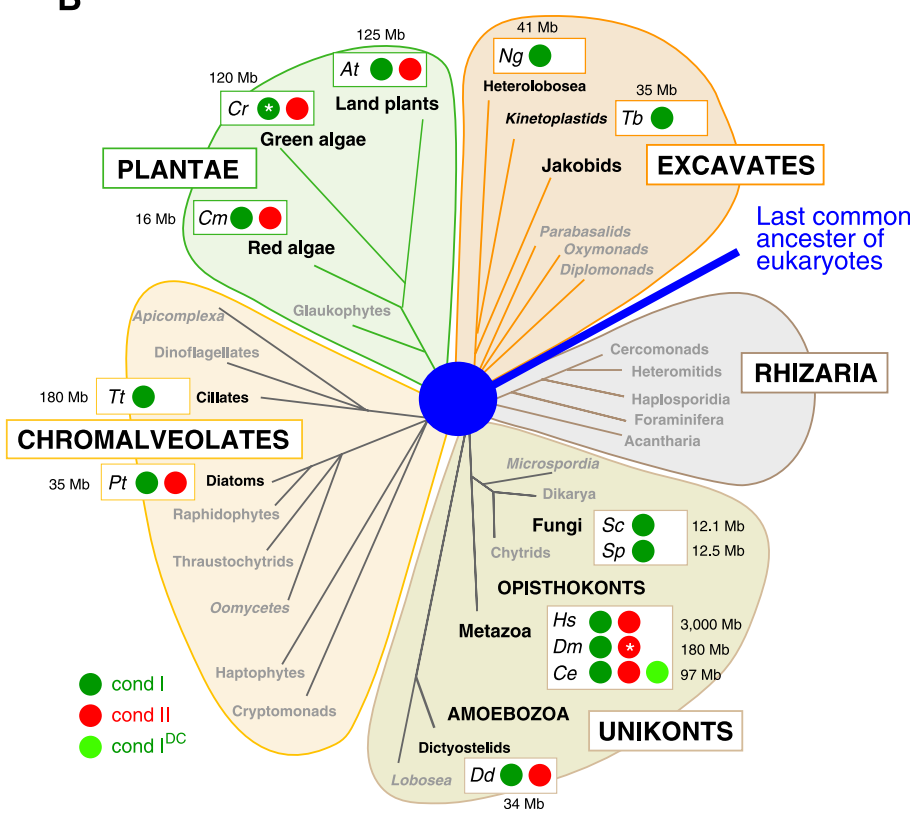

Figure 1. Molecular architecture and evolution of condensins. (A) Subunit composition of three different condensin complexes. Condensin I (left) and condensin II (center) share the same pair of SMC2 and SMC4 as their core subunits. The SMC dimer has a characteristic V shape with two ATP-binding "head" domains and a "hinge" domain responsible for dimerization. Each of the three non-SMC subunits of condensin I has a distantly related counterpart in those of condensin II. The CAP-H and CAP-H2 subunits belong to the kleisin family of proteins, whereas the CAP-D2, CAP-G, CAP-D3, and CAP-G2 subunits contain HEAT repeats. (Right) C. elegans has a condensin I-like complex (condensin $\mathrm{I}^{\mathrm{DC}}$ ) that participates in dosage compensation. Condensin $\mathrm{I}^{\mathrm{DC}}$ differs from canonical condensin I by only one subunit: DPY-27, an SMC4 variant (SMC4V), replaces SMC4 in condensin $\mathrm{I}^{\mathrm{DC}}$. (B) Phylogenetic tree of condensins in Eukaryota. Unikonts: (Hs) Homo sapiens (human); (Dm) D. melanogaster (fruit fly); (Ce) C. elegans (nematode); (Sc) $S$. cerevisiae (budding yeast); $(S p) S$. pombe (fission yeast); $(D d)$ Dictyostelium discoideum (slime mold). Chromalveolates: (Tt) T. thermophila (ciliate); (Pt) Phaeodactylum tricornutum (diatom). Plantae: (At) A. thaliana (green plant); (Cr) Chlamydomonas reinhardtii (green algae); (Cm) Cyanidioschyzon merolae (red algae). Excavates: ( $N g$ ) Naegleria gruberi; (Tb) Trypanosoma brucei. The presence of condensins I and II in each species is indicated by the green and red circles, respectively (condensin $\mathrm{I}^{\mathrm{DC}}$ is indicated by the light-green circles). The asterisks are added when not all three non-SMC subunits (of condensin I or II) are found in the sequenced regions of each genome, and the numbers indicate the genome size of each species. The composition of the tree was adapted from Koonin (2010) with permission from Elsevier (๔ 2010). 
Table 1. Condensin subunits in eukaryotic model organisms

\begin{tabular}{|c|c|c|c|c|c|c|}
\hline Subunits & Vertebrates & $\begin{array}{c}D . \\
\text { melanogaster }\end{array}$ & $\begin{array}{c}C . \\
\text { elegans }\end{array}$ & $\begin{array}{c}S . \\
\text { cerevisiae }\end{array}$ & $\begin{array}{c}S . \\
\text { pombe }\end{array}$ & $\begin{array}{c}\text { A. } \\
\text { thaliana }\end{array}$ \\
\hline \multicolumn{7}{|c|}{ Core subunits (common to I and II) } \\
\hline SMC2 & CAP-E/SMC2 & SMC2 & MIX-1 & Smc2 & Cut14 & CAP-E1 and CAP-E2 \\
\hline SMC4 & CAP-C/SMC4 & SMC4/Gluon & SMC-4 (DPY-27) ${ }^{a}$ & Smc4 & Cut3 & CAP-C \\
\hline \multicolumn{7}{|c|}{ I-specific subunits } \\
\hline HEAT (IA) & CAP-D2 & CAP-D2 & DPY-28 & Ycs4 & Cnd1 & CAB72176 \\
\hline HEAT (IB) & CAP-G & CAP-G & CAPG-1 & Ycs5/Ycg1 & Cnd3 & BАВ08309 \\
\hline Kleisin (IC) & CAP-H & CAP-H/Barren & DPY-26 & Brn1 & Cnd2 & AAC25941 \\
\hline \multicolumn{7}{|c|}{ II-specific subunits } \\
\hline HEAT (IIA) & CAP-D3 & CAP-D3 & HCP-6 & - & - & At4g15890.1 \\
\hline HEAT (IIB) & CAP-G2 & $?$ & CAP-G2 & - & - & CAP-G2/HEB1 \\
\hline Kleisin (IIC) & CAP-H2/nessy & CAP-H2 & KLE-2 & - & - & CAP-H2/HEB2 \\
\hline
\end{tabular}

${ }^{\mathrm{a}}$ In C. elegans, the SMC-4 subunit of condensin I is replaced with its variant, DPY-27, to make condensin $\mathrm{I}^{\mathrm{DC}}$, which has a specialized function in dosage compensation.

tions are possibly highly dynamic, and the subunit stoichiometry in a single complex might be either (SMC/ $\operatorname{MukB})_{2}(\mathrm{ScpA} / \mathrm{MukF})_{2}(\mathrm{ScpB} / \mathrm{MukE})_{2}$ or $(\mathrm{SMC} / \mathrm{MukB})_{2}(\mathrm{ScpA} /$ $\mathrm{MukF})_{2}(\mathrm{ScpB} / \mathrm{MukE})_{4}$ (Hirano and Hirano 2004; Gloyd et al. 2007; Woo et al. 2009).

\section{Overview of essential functions}

Recent series of genetic and cell biological studies reveal that condensins I and II fulfill nonoverlapping functions in many aspects of chromosome biology. Intriguingly, however, the relative contribution of the two condensin complexes to mitotic chromosome assembly and segregation apparently varies among different eukaryotic species, as discussed below.

\section{Mammals}

Early studies employing siRNA-mediated gene knockdown in human tissue culture cells demonstrated that condensins I and II have differential contributions to mitotic chromosome architecture and segregation, implying that both complexes play essential roles in mitosis (Ono et al. 2003, 2004; Hirota et al. 2004). These studies also showed that the two condensin complexes display strikingly different behaviors during the cell cycle (Fig. 2A, from top to right). Condensin I is sequestered in the cytoplasm during interphase and gains access to chromosomes only after the nuclear envelope breaks down in prometaphase. In contrast, condensin II localizes to the nucleus from interphase through prophase and participates in an early stage of chromosome condensation within the prophase nucleus. After nuclear envelope breakdown (NEBD), condensins I and II collaborate to support proper assembly of chromosomes in which sister chromatids are well resolved by metaphase and to promote faithful segregation in anaphase. The characteristic behaviors of the two condensin complexes are most likely to be central to our understanding of their action and regulation. At the level of ontogeny, knocking out the gene encoding the CAP-G2 subunit in mice has been shown to cause embryonic lethality (Smith et al. 2004; Xu et al. 2006). Whereas knockout mice for the other subunits have not yet been described, all of them are anticipated to be essential for embryogenesis. Remarkably, recent studies started to uncover tissue-specific developmental defects caused by a hypomorphic mutation or misregulation of condensin subunits, which will be discussed in later sections.

\section{Chicken DT40 cells}

Generation of conditional knockout cell lines by using chicken DT40 cells offers an alternative method for silencing target genes of interest and is arguably superior to siRNA-mediated knockdown techniques widely used in mammalian cells. Such cell lines can be established by the introduction of a tetracycline-regulated transgene, followed by disruption of the authentic loci through homologous recombination. Hudson et al. (2003) applied this technique to the condensin core subunit SMC2 (also known as ScII) and showed that it is required for nonhistone protein assembly and structural integrity of mitotic chromosomes. A subsequent mutational study demonstrated that ATP binding, but not hydrolysis, by SMC2 is required for its stable association with chromosomes (Hudson et al. 2008). In DT40 cells depleted of SMC2, defects in chromosome condensation are relatively modest in metaphase but become very severe in anaphase, leading to the proposal that a hypothetical factor termed regulator of chromosome architecture (RCA) might cooperate with condensins to preserve the characteristic shape of metaphase chromosomes (Vagnarelli et al. 2006).

\section{Xenopus laevis (cell-free egg extracts)}

Metaphase chromosomes can be reconstituted in vitro starting from simple substrates, such as sperm chromatin, in Xenopus cell-free egg extracts. Biochemical characterization of the chromosomes assembled in the cellfree extracts led to the discovery of the first condensin complex, now known as condensin I (Hirano and Mitchison 1994; Hirano et al. 1997). A subsequent study showed that condensin II is also present in the cell-free extracts, albeit as a less abundant component compared with 
A

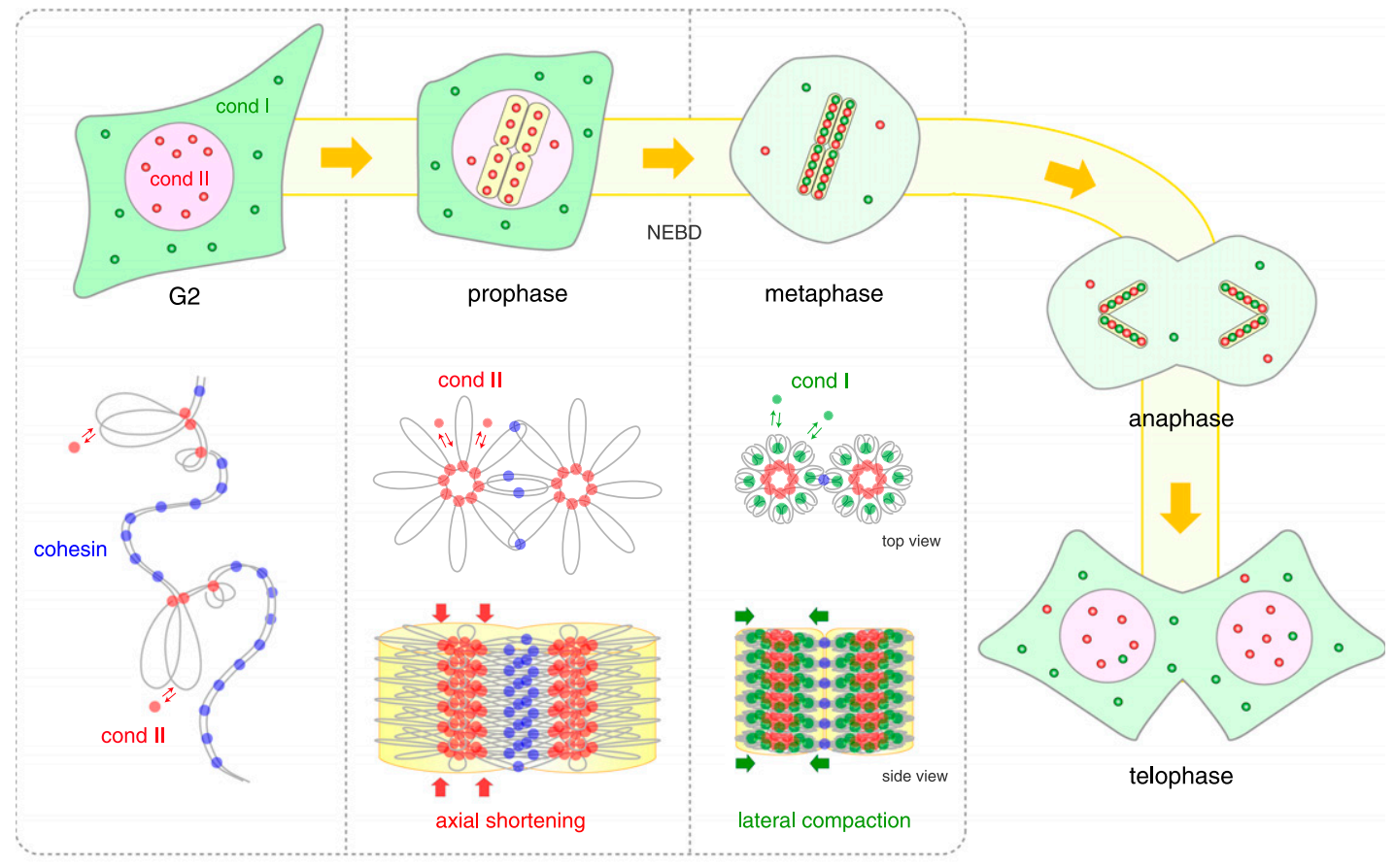

B

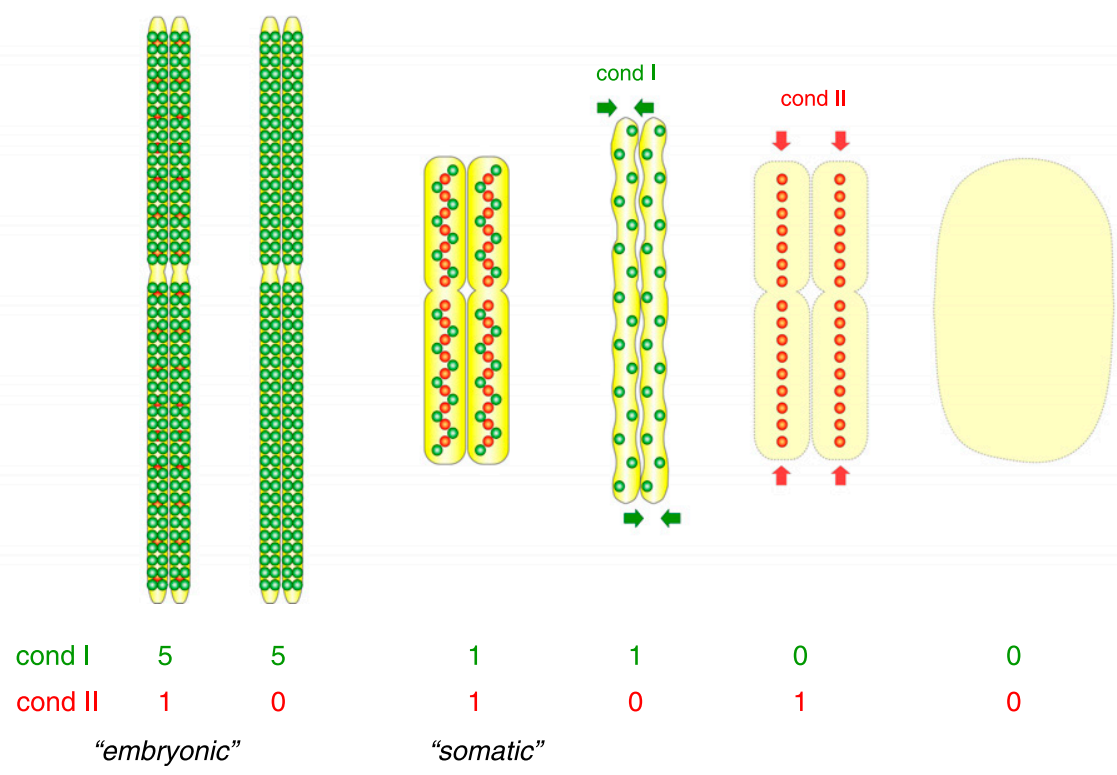

Figure 2. Sequential and balancing actions of condensins I and II. (A) Cell cycle dynamics of condensins I and II in mammalian cells (shown from top to right). During G2 phase, condensin II localizes to the nucleus, whereas condensin I is sequestered in the cytoplasm. (G2, bottom) Although little is known about what condensin II does at this stage of the cell cycle, it may counteract cohesin to prepare for mitosis. For simplicity, catenation between sister DNAs is not shown. In prophase, condensin II participates in the early stage of chromosome condensation within the nucleus. After nuclear envelope breakdown (NEBD), condensin I gains access to chromosomes and collaborates with condensin II to assemble fully resolved sister chromatids by metaphase. It has been proposed that condensin II primarily contributes to axial shortening of chromatids (prophase, bottom), whereas condensin I contributes to lateral compaction (metaphase, bottom). How the differential distribution of the two complexes might be re-established upon nuclear envelope assembly at telophase is unknown. Cohesin, condensin I, and condensin II are indicated by the blue, green, and red circles, respectively. The cartoons presented here are intended to emphasize the proposed, differential contributions of condensins I and II to chromosome assembly and are therefore admittedly oversimplified. Clearly, the two condensin complexes do not act in a completely step-wise fashion: Condensin II continues to act in concert with condensin I even after NEBD. (B) A conceptual model of how the balancing actions of condensins I and II might determine chromosome shapes. "Embryonic" chromosomes with a condensin I:II ratio of $\sim 5: 1$ are long and thin because condensin I's contribution is predominant. In contrast, "somatic" chromosomes with a ratio of $\sim 1: 1$ are short and thick. Further depletion of condensin II from this condition lengthens the chromosomes (1:0), whereas depletion of condensin I widens them (0:1). In the absence of both condensins I and II, only cloud-like, fuzzy masses of chromatin are observed (0:0). Condensins I and II are indicated by the green and red circles, respectively. 
condensin I (Ono et al. 2003). In accordance with the relative ratio between the two complexes (condensin I:condensin II $=\sim 5: 1$, condensin I plays a predominant role in chromosome assembly, whereas condensin II has a minor contribution to this process in this cell-free extract. As discussed later in this review, a more recent study using this experimental system has demonstrated that the relative ratio of condensin I to II indeed acts as a critical factor that determines the shape of mitotic chromosomes (Shintomi and Hirano 2011).

\section{Drosophila melanogaster}

Early genetic studies in Drosophila showed that proper assembly and segregation of mitotic chromosomes require condensin I subunits, including SMC4/Gluon (Steffensen et al. 2001), CAP-H/Barren (Bhat et al. 1996), CAP-G (Dej et al. 2004), and CAP-D2 (Savvidou et al. 2005). It has also been shown that condensin I contributes to proper localization of topoisomerase II (topo II) to the chromosome axis (Coelho et al. 2003) and maintenance of the structural integrity of centromeric heterochromatin (Oliveira et al. 2005). It remains to be determined whether the fivesubunit condensin II complex is present in Drosophila because the gene encoding CAP-G2 has not yet been found in the sequenced region of the Drosophila genome. Currently available evidence suggests that the putative condensin II subunits (CAP-D3 and CAP-H2) may not play a major role in mitotic chromosome organization and segregation (Savvidou et al. 2005; Oliveira et al. 2007) and that they have meiotic functions instead, making a crucial contribution to anaphase I chromosome segregation (Hartl et al. 2008b; see below).

\section{Caenorhabditis elegans}

Unlike other eukaryotes, C. elegans has three condensin complexes (Csankovszki et al. 2009a). In addition to condensins I and II, this organism possesses a condensin I-like complex (known as condensin $\mathrm{I}^{\mathrm{DC}}$ ) that constitutes a part of the large protein complex involved in dosage compensation. Condensin $\mathrm{I}^{\mathrm{DC}}$ differs from the canonical condensin I complex by only one subunit (Fig. 1A; Table 1). It is assumed that duplication of SMC4 during evolution gave rise to DPY-27, leading to the creation of condensin $\mathrm{I}^{\mathrm{DC}}$ with a specialized function of chromosome-wide gene repression. Notably, the mitotic phenotypes observed in condensin I-deficient cells are far less severe than those in condensin II-deficient cells, implying that condensin II plays a dominant role during mitosis in this organism. This atypical observation could be related to the fact that C. elegans has a unique holocentric structure in which centromeres/kinetochores assemble and function along the entire chromosome arms (Hagstrom et al. 2002).

\section{Fungi}

None of the genes encoding condensin II-specific subunits can be found in the genome of fungi. In S. pombe, the five-subunit condensin I complex localizes to the cytoplasm during interphase and relocates to the nucleus in mitosis to support chromosome condensation and segregation (Sutani et al. 1999). Unlike in S. pombe, the condensin subunits localize to the nucleus throughout the cell cycle in S. cerevisiae (Freeman et al. 2000). Although this behavior is reminiscent of that of condensin II in human cells, the amino acid sequences of the subunits of $S$. cerevisiae condensin clearly indicate that it is condensin I. The constitutive nuclear localization of condensin I in S. cerevisiae could be related to its rather peculiar cell cycle in which S-phase and M-phase events partially overlap with each other (e.g., the spindle begins to form in late S phase, and there is no clear G2 phase). Whereas many early studies on $S$. cerevisiae condensin focused on its role in promoting the condensation and segregation of rDNA (e.g., Freeman et al. 2000; Lavoie et al. 2004), more recent, state-of-the-art imaging techniques have uncovered the function of condensin $I$ as a chromatin "spring" at pericentromeric regions in metaphase (Stephens et al. 2011) and its contribution to "recoiling" of stretched chromosome arms in anaphase (Renshaw et al. 2010).

\section{Arabidopsis thaliana}

The genome of $A$. thaliana encodes all subunits of condensins I and II (Hirano 2005). Whereas the A. thaliana genome has a single essential gene $(A t C A P-C)$ encoding SMC4 (Siddiqui et al. 2006), it possesses two paralogous genes for SMC2, known as AtCAP-E1 and AtCAPE2 (Siddiqui et al. 2003). The two SMC2 proteins are functionally redundant: Single-homozygous mutants $\left(E 1^{-/-}\right.$and $\left.E 2^{-I-}\right)$ are viable, whereas double-homozygous $\left(E 1^{-/-} E 2^{-/-}\right)$and $E 1^{-/-} E 2^{+/-}$plants are both embryoniclethal. On the other hand, an $E 1^{+/-} E 2^{-/-}$plant results in meristem disorganization and fasciation, indicating that SMC2 activity above a certain threshold is required for proper development of $A$. thaliana. Very recently, a genetic screen for mutants that exhibit hypersensitivity to excess boron has led to the somewhat surprising conclusion that condensin II is nonessential for mitosis and instead plays a role in alleviating DNA damage (Sakamoto et al. 2011). Whereas genetic studies have yet to be reported for the genes encoding condensin I-specific subunits, it is anticipated that condensin I is sufficient for mitotic chromosome assembly and segregation in this organism.

\section{Tetrahymena thermophila}

T. thermophila is a ciliate that maintains two nuclei, a germline micronucleus and a somatic macronucleus, in a cell. The micronucleus divides by mitosis in a manner similar to that observed in many other eukaryotes. On the other hand, the formation and division of the macronucleus is highly unique: It is derived from the micronucleus through genomic rearrangement, contains $>200$ chromosome fragments ranging in size from 20 to $>3000 \mathrm{~kb}$, and divides by a poorly characterized mechanism of "amitosis" (Yao and Chao 2005). The macronucleus genome of T. thermophila encodes SMC2, SMC4, and all condensin I-specific subunits but lacks the genes for condensin II-specific subunits (Eisen et al. 2006). 
Somatic gene knockdown of SMC4 causes defects in amitotic division of the macronucleus, thereby compromising vegetative growth of $T$. thermophila (Cervantes et al. 2006). Notably, the T. thermophila genome possesses multiple genes for CAP-D2 and CAP-H, a situation rarely found in other eukaryotic genomes. It will be of great interest in the future to test whether distinct forms of the condensin I complex may exist in this organism and localize differentially to the micronucleus and micronucleus.

\section{Bacteria}

Although numerous studies have provided evidence that SMC-ScpAB and MukBEF play an important role in chromosome compaction and segregation in bacterial cells, exactly how the bacterial condensin complexes might work remains poorly understood /Graumann and Knust 2009). Recent studies in Bacillus subtilis have shown that SMC is recruited to centromere-like sequences (termed parS) near the replication origin in a manner dependent on ParB/SpoOJ (Gruber and Errington 2009; Sullivan et al. 2009). It is hypothesized that recruitment of SMC-ScpAB to the origin region promotes efficient chromosome segregation by compacting replicated DNAs as they emerge from the replisome located at mid-cell. Consistent with this idea, a recent analysis employing chromosome conformation capture carbon copy (5C) has shown that the parS sites have a great impact on global orientation, compaction, and segregation of chromosomes in Caulobacter crescentus (Umbarger et al. 2011). Moreover, expression of a dominant-negative form of SMC allows replication of the parS sites yet interferes with their segregation (Schwartz and Shapiro 2011).

\section{How might condensins I and II collaborate to assemble chromosomes?}

As discussed above, most eukaryotic species have two different condensin complexes, and, at least in vertebrates, both of them appear to play essential roles in mitotic chromosome assembly and segregation. A number of fundamental questions then arise. How are the two condensin complexes regulated differentially during the cell cycle? How are their functions coordinated with each other during the process of chromosome assembly?

\section{Sequential actions of condensins $I$ and II}

Perhaps one of the most intriguing observations made in early studies using HeLa cells was that condensins I and II display strikingly different localization during interphase: Condensin I is cytoplasmic, whereas condensin II is nuclear (Fig. 2A, G2; Hirota et al. 2004; Ono et al. 2004; Gerlich et al. 2006). Importantly, the differential distribution of condensins I and II in interphase can faithfully be recapitulated in the Xenopus cell-free egg extracts (Shintomi and Hirano 2011). Moreover, even in meiotic cells such as mouse oocytes, condensin II is present within the germinal vesicle (i.e., the large nucleus found in fully grown oocytes), whereas condensin I is sequestered in the cytoplasm (Lee et al. 2011). Thus, the characteristic localization of the two condensin complexes appears to be a fundamental feature highly conserved among many eukaryotic species and cell types.

What, then, is the physiological significance of this spatial regulation? There are several possible scenarios. For instance, condensin II plays an important role in the interphase nucleus and therefore needs to stay within the nucleus after mitosis. On the other hand, condensin I has a minimal function during interphase or is even toxic if it stays in the nucleus and therefore needs to be actively transported out of the nucleus. An alternative possibility is that the sequestration of condensin I in the cytoplasm until NEBD may act as a mechanism that determines the order of actions of the two condensin complexes, thereby ensuring the two-step process of mitotic chromosome condensation (Hirano 2005; Marko 2008). In fact, it seems natural that condensin II initiates the first step of condensation during prophase because it is already present within the nucleus (Fig. 2A, prophase; Hirota et al. 2004; Ono et al. 2004). The second step is triggered when condensin I gains access to chromosomes after NEBD in prometaphase and is completed by coordinated actions of condensins I and II in metaphase (Fig. 2A, metaphase). Consistent with this scenario, whereas both complexes are concentrated in the axial region of metaphase chromatids, condensin I signals tend to be found outside of condensin II signals (Ono et al. 2003). Moreover, as judged by fluorescence recovery after photobleaching (FRAP), condensin I interacts with chromosomes in a more dynamic manner than condensin II does (Gerlich et al. 2006).

\section{Balancing the actions of condensins I and II}

An early study noticed that the relative abundance between condensins I and II might vary among different cell types and that this simple parameter might directly reflect their functional contribution to chromosome assembly in a given cell type (Ono et al. 2003). For instance, in Xenopus egg extracts where the ratio of condensin I to II is estimated to be $\sim 5: 1$, condensin I's function is predominant. On the other hand, in HeLa cells with an $\sim 1: 1$ ratio, the functional contribution of the two complexes is apparently equal (Fig. 2B). Also intriguing is the observed tendency of embryonic chromosomes assembled in Xenopus egg extracts to be long and thin, whereas somatic chromosomes observed in HeLa cells are short and thick. To directly test the hypothesis that the balancing actions of the two condensin complexes might determine the shape of metaphase chromosomes, a recent study manipulated the level of condensins I and II by quantitative immunodepletion in Xenopus egg extracts (Shintomi and Hirano 2011). Remarkably, when the relative ratio of condensin I to II was forced to be smaller (from $\sim 5: 1$ to $\sim 1: 1$ ), the embryonic chromosomes became shorter and thicker. When condensin II was depleted further to make an extract with a 1:0 ratio, the chromosomes got longer. These results strongly suggest 
that condensin II's action primarily contributes to axial shortening of chromatids, whereas condensin I supports their lateral compaction (Fig. 2A,B).

This idea has gained additional support from recent studies in vivo. First, when cells are arrested at metaphase for a long period, condensin I is inactivated through caspase-dependent cleavage, resulting in the formation of short and thick chromosomes (Lai et al. 2010). Second, abnormally short and wavy chromosomes are observed when condensin II is hyperactivated in cells deficient in its negative regulator, MCPH1 (Yamashita et al. 2011). Third, a more recent study has depleted condensin I- or IIspecific subunits from chicken DT40 cells and has shown that condensin II is required for establishing rigid chromosome axes whereas condensin I helps organize chromatin loops around the axes (Green et al. 2012).

\section{Toward mechanistic understanding of the assembly} and shaping of chromosome arms

Although the recent studies discussed above start to shed new light on a previously underappreciated set of questions, it remains unclear mechanistically how the sequential and balancing actions of condensins I and II might support chromosome assembly and shaping. Future experiments should critically test what would happen if the order of actions were reversed experimentally, for example. It will also be of great importance to understand why the shape of metaphase chromosomes might change during development and vary among different cell types. Conceivably, the ratio of condensin I to II would not be the sole parameter that affects chromosome shaping. Its relationships with other activities, such as gene expression and replication programs, need to be explored rigorously. Finally and most importantly, an indepth comparison of biochemical activities associated with condensins I and II would be vital in fully understanding the mechanistic basis of chromosome assembly and shaping.

The outcome from these efforts should eventually be integrated with structural studies of chromosomes, which themselves have a long history yet remain controversial (for review, see Belmont 2006; Marko 2008). For example, on the basis of the visualization of early phases of chromosome condensation by light and electron microscopy, one study proposed a hierarchical folding, axial glue model (Kireeva et al. 2004). According to this model, condensation starts with progressive folding of a $30-\mathrm{nm}$ fiber into fibers with increasing diameters by middle prophase. The next step involves large-scale coiling of a 200- to 250-nm fiber in late prophase, followed by stabilization by "glue" distributing along the chromatid axis in metaphase. On the other hand, another study using cryoelectron microscopy and X-ray scattering does not support the existence of the 30-nm fiber in mitotic chromosomes, arguing that chromosomes are primarily composed of irregularly folded nucleosome fibers (Nishino et al. 2012). Despite the apparent differences, the two models share the common view that condensins become enriched at the chromatid axis and thereby play crucial roles in establishing rod-shaped chromatids observed in metaphase. From a biochemical point of view, it is important to note that metaphase chromosomes, when isolated with great caution, have a surprisingly simple protein composition (Gasser and Laemmli 1987; Hirano and Mitchison 1994). Therefore, seemingly bold attempts to reconstitute a "chromosome" from purified components would not be completely unrealistic, possibly offering a powerful approach to filling the gap that currently exists between the biochemical and structural studies of chromosomes. Other important lines of experimental approaches would include state-of-the-art microscopic observations of chromosomes in living cells (MoraBermudez et al. 2007; Renshaw et al. 2010) and mechanical and enzymatic manipulations of native chromosomes in vitro (Pope et al. 2006; Kawamura et al. 2010).

\section{Roles of condensins I and II in centromere/kinetochore organization}

Condensins play crucial roles in the structural and functional organization of the centromere/kinetochore region of chromosomes. In animal cells, for instance, condensin I (but not condensin II) regulates the stiffness of centromeric heterochromatin, the loss of which loosens centromeric cohesion and leads to abnormal orientations and/or movements of sister kinetochores (Ono et al. 2004; Oliveira et al. 2005; Gerlich et al. 2006; Ribeiro et al. 2009). In S. cerevisiae, condensin is responsible for axial compaction of pericentric chromatin and acts as part of a molecular "spring" that generates tension between bioriented sister centromeres (Stephens et al. 2011).

C. elegans has unique chromosome structures, known as holocentric chromosomes, in which numerous centromeres/kinetochores assemble along the entire length of each chromatid. In this organism, although condensin I distributes to entire chromosomes (Csankovszki et al. 2009a), condensin II is largely confined to the centromeres, and its mutation causes massive merotelic attachments and chromosome segregation defects (Stear and Roth 2002). Interestingly, a subfraction of condensin II is also found to be enriched at or near the inner kinetochore plate on monocentric chromosomes in humans (Ono et al. 2004), Xenopus (Shintomi and Hirano 2011), and Drosophila (Savvidou et al. 2005). To what extent condensin II might contribute to the assembly and structural maintenance of kinetochores remains unclear. For instance, although kinetochores are apparently normal both structurally and functionally in SMC2-depleted DT40 cells (Ribeiro et al. 2009), a recent study using Xenopus egg extracts reports that condensin II is required for efficient loading of CENP-A and contributes to retention of CENP-A nucleosomes at centromeres (Bernad et al. 2011).

\section{Cell cycle regulators of condensins}

Given the multisubunit architecture of condensins and their diverse functions in chromosome dynamics through- 
out the cell cycle, it is anticipated that a wide variety of regulatory signals are imposed on condensin subunits to control their subcellular localization, chromosomal loading/unloading, activation/inactivation, and fine-tuning of all these events. It seems clear, however, that even the most updated list of their phosphorylation sites, created by modern phosphoproteomics approaches, remains incomplete (Nousiainen et al. 2006; Hegemann et al. 2011; Pagliuca et al. 2011). On the other hand, multiple mitotic kinases such as cyclin-dependent kinase 1 (Cdk1), aurora $\mathrm{B}$, and polo-like kinases (Plks) have been shown to phosphorylate and regulate condensins, although their relative contributions apparently vary among different organisms.

\section{Cdk1}

Condensin I purified from Xenopus egg extracts displays an ability to introduce positive superhelical tension into dsDNA in vitro (Kimura and Hirano 1997). This and other related activities are stimulated by Cdk1-cyclin B-dependent phosphorylation of the regulatory subunits (Kimura et al. 1998, 1999), implying that these activities might represent physiologically relevant ones that help promote mitotic chromosome condensation in vivo. Evidence is also available that in $S$. pombe, Cdk 1 phosphorylation of the SMC4/Cut3 subunit promotes condensin's entry into the nucleus during mitosis (Sutani et al. 1999). A recent study in HeLa cells has shown that Cdk1 phosphorylates the CAP-D3 subunit of condensin II at Thr 1415 and thereby promotes the early stage of chromosome condensation (Abe et al. 2011). It will be of great importance to understand precisely how different combinations of Cdks and mitotic cyclins might regulate the spatiotemporal activation of condensins I and II. It is possible, for instance, that Cdk1/2-cyclin A and Cdk1cyclin B sequentially phosphorylate condensin II to coordinate the early and late stages of chromosome condensation (Hirano 2005). On the basis of a quantitative proteomics approach, Pagliuca et al. (2011) have recently proposed that a class of Cdk substrates might interact first with cyclin A and later with cyclin B through the socalled "handover" mechanism. Condensin II is indeed an excellent candidate for such Cdk substrates, although direct evidence supporting this idea remains to be obtained.

\section{Aurora B}

An early study reported that siRNA-mediated depletion of aurora B from Drosophila S2 cells causes a drastic decrease of a condensin I subunit associated with mitotic chromosomes (Giet and Glover 2001). However, the extent of the functional contribution of aurora B to chromosomal loading of condensins appears to vary among different species or cell types. For example, depletion of aurora B from Xenopus egg extracts barely or only subtly affects the level of condensin I loaded onto metaphase chromosomes and the resulting chromosome morphology (MacCallum et al. 2002; Takemoto et al. 2007). In HeLa cells, inhibition or depletion of aurora $\mathrm{B}$ reduces the amount of condensin I, but not of condensin II, loaded onto chromosomes in prometaphase (Lipp et al. 2007). Conceivably, aurora B's impact on condensin I would be maximal in anaphase when an additional amount of condensin I becomes associated with the chromosome arms (Gerlich et al. 2006; Tada et al. 2011). Evidence is also available that aurora B might have a role in anaphase chromatid contraction through a mechanism independent of condensins (Mora-Bermudez et al. 2007). In C. elegans, a requirement for aurora B in condensin I loading appears much more severe than in vertebrate cells (Hagstrom et al. 2002). Although bulk loading of condensin II is barely affected in the absence of aurora B, its localization at kinetochores appears compromised (Collette et al. 2011), as has been reported previously in HeLa cells (Ono et al. 2004). In S. cerevisiae, it was proposed that mitotic folding of the rDNA repeat involves two steps: an aurora B/Ipl1independent step from G2 through metaphase and an aurora B/Ipl1-dependent step in anaphase (Lavoie et al. 2004). In S. pombe, aurora B/Ark1 phosphorylates three specific residues at the $\mathrm{N}$-terminal domain of CAP-H/ Cnd2, a condensin I subunit, to support proper chromosome segregation (Nakazawa et al. 2011), possibly by modulating condensin-chromatin interactions (Tada et al. 2011).

\section{Plks}

A recent study has shown that in S. cerevisiae, the major kinase that phosphorylates condensin subunits in anaphase is in fact Polo/Cdc5, rather than aurora B/Ipl1 (StPierre et al. 2009). Polo/Cdc5-mediated phosophorylation causes hyperactivation of condensin's supercoiling activity in vitro and is required for proper anaphase condensation in vivo. It is very important to note, however, that Polo/Cdc5-mediated phosphorylation alone is insufficient to activate the supercoiling activity and must act in concert with Cdk1-mediated phosphorylation. Possible cooperative actions of Polo/Plk1 and Cdk1 in the regulation of condensin II have been suggested in a recent study using HeLa cells (Abe et al. 2011). Thus, an emerging view is that a single dominant kinase is not enough to regulate the highly complex and dynamic actions of condensins in any organisms (Bazile et al. 2010). It will be important in the future to fully understand how multiple phosphorylation events catalyzed by the mitotic kinases help coordinate the spatiotemporal loading and activation of condensins in each species.

\section{Other positive and negative regulators}

In addition to the mitotic kinases discussed above, a series of recent studies has identified other positive and negative regulators of condensins. For example, casein kinase 2 (CK2) phosphorylates condensin I and negatively regulates its functions during interphase (Takemoto et al. 2006). Notably, CK2-mediated phosphorylation of condensin I reduces its DNA supercoiling activity in vitro. According to another study, protein phosphatase $2 \mathrm{~A}$ (PP2A) facilitates chromosomal recruitment of condensin II in Xenopus egg extracts as well as in human cells in a manner independent of its own catalytic activity 
(Takemoto et al. 2009). Moreover, the monomethylation of histone $\mathrm{H} 4$ at Lys 20 (H4K20me1) has been reported to play a role in recruiting condensin II to prophase chromosomes (Liu et al. 2010). Finally, it has been shown that MCPH1, a BRCA1 C-terminal (BRCT) domaincontaining protein responsible for primary microcephaly, acts as a highly specific, potent inhibitor of condensin II and helps prevent premature condensation of chromosomes during G2 phase (Yamashita et al. 2011). Its potential involvement in the etiology of microcephaly and in brain development are discussed below.

\section{Gene regulation and other chromosomal functions}

Accumulating lines of evidence demonstrate that condensins participate in a wide variety of nonmitotic chromosome functions in many different model organisms. Such examples are as diverse as gene regulation (transvection and dosage compensation), recombination (rDNA repeat maintenance), DNA damage response, and repair.

\section{Polytene disassembly and transvection control in Drosophila}

Polytene chromosomes are gigantic chromosomes in which thousands of chromatids are aligned in precise register and are found in some specialized cells. In Drosophila, for example, ovarian nurse cells assemble polytene chromosomes, which are then disassembled into unpaired homologs and chromatid fibers during midoogenesis. A recent genetic study has shown that two genes encoding putative subunits of condensin II, Cap$D 3$ and $\mathrm{Cap}-\mathrm{H} 2$, are required for the disassembly of these polytene chromosomes (Fig. 3A; Hartl et al. 2008a). Consistently, overexpression of Cap-H2 in the larval salivary glands induces drastic disassembly of their polytene chromosomes. Equally important, the same study has provided evidence that condensin II negatively regulates transvection, a process in which certain mutant alleles are transcriptionally influenced through association with their homologous locus (Fig. 3B). These results strongly suggest that the ability of condensin II to separate (or "resolve") chromatids is not limited during mitosis; rather, it promotes disruption of chromatidchromatid interactions within polytene chromosomes and also restricts homolog-homolog interactions in diploid somatic nuclei.

\section{Dosage compensation in C. elegans}

Dosage compensation is the process that equalizes the level of X-linked gene expression between the sexes in animals. In C. elegans, a large protein complex called the dosage compensation complex (DCC) binds to both X chromosomes in hermaphrodites to down-regulate gene expression by half, thereby equalizing X-linked gene expression levels with those in males (Fig. 3C; Csankovszki et al. 2009b; Ercan and Lieb 2009). The DCC is composed of a condensin I-like complex (known as condensin $\mathrm{I}^{\mathrm{DC}}$ ) and another five-subunit complex containing SDC-1, SDC-2, SDC-3, DPY-21, and DPY-30. Experiments combining genome-wide chromatin precipitation assays have revealed that the DCC is enriched at $\sim 1500$ sites scattered along the length of the $\mathrm{X}$ chromosomes that can be categorized into two different classes: rex (recruiting element on $\mathrm{X}$ ) sites and dox (dependent on $\mathrm{X}$ ) sites (McDonel et al. 2006; Ercan et al. 2007; Blauwkamp and Csankovszki 2009; Jans et al. 2009). The rex sites contain a 12-base-pair sequence motif called MEX (motif enriched on $\mathrm{X}$ ) and are able to recruit the DCC when detached from the X. On the other hand, the dox sites are bound by the DCC only when they are located on the X chromosome

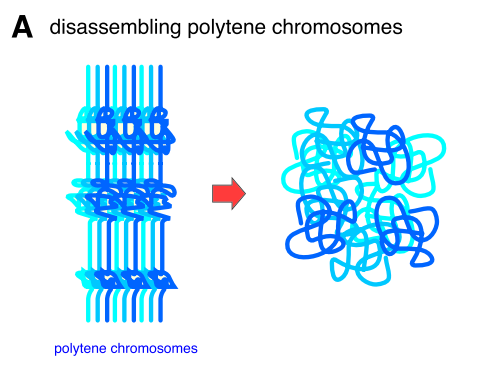

B antagonizing transvesion

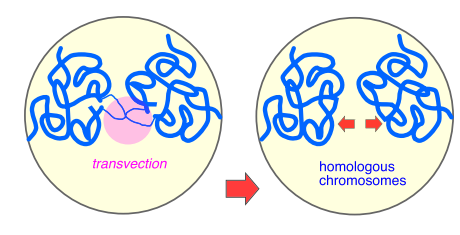

C regulating dosage compensation

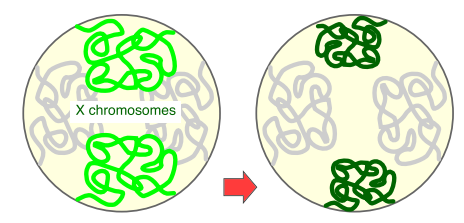

D stablizing rDNA repeat

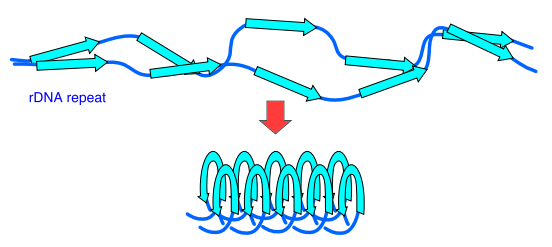

E clustering tRNA genes

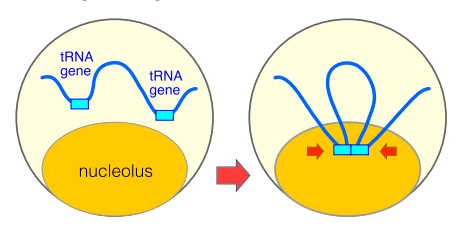

Figure 3. A diverse array of interphase chromosomal functions supported by condensins. $(A)$ Condensin II promotes the disassembly of polytene chromosomes in D. melanogaster. (B) Condensin II antagonizes transvection in $D$. melanogaster, possibly by restricting physical interactions between homologous chromosomes. $(C)$ In C. elegans hermaphrodites, condensin $\mathrm{I}^{\mathrm{DC}}$ associates with both $\mathrm{X}$ chromosomes and down-regulates expression of $\mathrm{X}$-linked genes by half, possibly by changing higher-order chromosome structures of the X. (D) S. cerevisiae condensin plays a crucial role in stabilizing the rDNA repeat. $(E) S$. cerevisiae condensin promotes clustering (and subsequent nucleolar localization) of tRNA genes that are scattered along its genome. 
(Jans et al. 2009). It will be important to understand how the DCC might initially be recruited to some of these sites and how it might potentially use a "spreading" mechanism to distribute along the length of the X chromosome (Ercan et al. 2009).

The difference between condensin I and condensin $\mathrm{I}^{\mathrm{DC}}$ resides in only one subunit (Fig. 1A; Table 1; Csankovszki et al. 2009a). It remains unknown how such a singlesubunit substitution might alter the original molecular activity of condensin $\mathrm{I}$, thereby allowing condensin $\mathrm{I}^{\mathrm{DC}}$ to participate in chromosome-wide gene repression. Intriguingly, a recent study suggests that the DCC may not repress genes by direct binding; rather, the DCC may act at a distance, possibly by bringing dosage-compensated genes to the vicinity of DCC-bound sites (Jans et al. 2009). Another study provided evidence that the histone H2A variant H2A.Z might function in dosage compensation through a mechanism that helps restrict the DCC to the X chromosome (Petty et al. 2009). Thus, information from these studies on condensin $\mathrm{I}^{\mathrm{DC}}$ should provide a paradigm that will readily be applicable to our understanding of the general mechanisms of action of condensins.

\section{Maintenance of the rDNA repeat in S. cerevisiae}

In $S$. cerevisiae, $\sim 200$ copies of rDNA are clustered in a long tandem array on chromosome XII. A single unit of rDNA ( $\sim 9.1 \mathrm{~kb}$ long) comprises a 35S rRNA-coding sequence (transcribed by RNA polymerase I [Pol I]), a 5S rRNA-coding sequence (transcribed by RNA polymerase III [Pol III]), and two nontranscribed spacers (NTS1 and NTS2). Fob1, which binds to the replication fork barrier (RFB) sequence located in NTS1, is known to contribute to the maintenance of rDNA copy number by stimulating recombination. Johzuka et al. (2006) set up a genetic screen for gene products that, in concert with Fob1, are required for preventing contraction of the rDNA repeat and identified three subunits of budding yeast condensin. Condensin is recruited to the RFB in a Fob1-dependent manner, and this recruitment requires three additional gene products: Tof2, Csm1, and Lrs4 (Johzuka and Horiuchi 2009). Whereas condensin also associates with the 35S rRNA-coding region, this association is swept away by Pol I-dependent transcription in fob1 mutant cells. Conversely, inactivation of Pol I suppresses the drastic contraction of the rDNA repeat in a fob1-condensin double mutant (Johzuka and Horiuchi 2007). These results strongly suggest not only that condensin plays a critical role in maintaining the copy number of the rDNA repeat (Fig. 3D), but that condensin's association with chromatin is incompatible with active transcription.

Two recent studies further pointed out the counteraction between transcription and condensin functions. First, the protein phosphatase Cdc14 inhibits Pol I transcription during mitosis, which in turn allows condensin's association with rDNA and thereby leads to proper condensation and segregation (Clemente-Blanco et al. 2009). Second, in a strain with a low rDNA copy number, the average rate of rDNA transcription per copy increases, leading to the loss of condensin association from this locus. Under this condition, pairing between the sister rDNA loci is weakened, and the cells become sensitive to DNA damage, presumably due to compromised recombinational repair (Ide et al. 2010). Thus, the rDNA repeat in $S$. cerevisiae represents a busy crossroad where a number of chromosomal activities (e.g., replication, transcription, recombination, and segregation) meet and it will continue to act as an excellent model system for dissecting the multilayered functions of condensin. The observation that nutrient starvation promotes condensin loading onto the rDNA repeat to maintain its stability is another example of promising future studies (Tsang et al. 2007). In S. pombe, some nucleolar proteins have been found to affect condensin's accumulation at the rDNA region (Nakazawa et al. 2008).

\section{Differential clustering of tRNA genes in S. cerevisiae and $\mathrm{S}$. pombe}

Although as many as $\sim 270$ tRNA genes are scattered in the $S$. cerevisiae genome, most of them are clustered and located at the nucleolus at the cytological level (Fig. 3E; Thompson et al. 2003). A recent study has shown that clustering of tRNA genes requires condensin functions and that condensin indeed binds to active tRNA genes, as judged by a chromatin immunoprecipitation (ChIP) assay (Haeusler et al. 2008). Disruption of microtubules releases tRNA genes from the nucleolus without dispersing the clusters themselves, indicating that clustering of tRNA genes and their nucleolar localization are separable processes. An independent, genome-wide ChIP-chip analysis has provided a complementary picture by demonstrating that budding yeast condensin associates with tRNA genes and other sites bound by TFIIIC, a component of the Pol III transcription machinery ( $\mathrm{D}^{\prime}$ Ambrosio et al. 2008b). It is therefore possible that TFIIIC is responsible for specifying a class of condensin-binding sites in the budding yeast genome.

Notably, TFIIIC is also implicated in large-scale chromosome organization in $S$. pombe, yet in a way different from that in S. cerevisiae. In S. pombe, a fraction of TFIIIC with Pol III at tRNA genes is clustered near centromeres, whereas another fraction of TFIIIC without Pol III is concentrated in several distinct bodies at the nuclear periphery, referred to as chromosome-organizing clamps (COCs) (Noma et al. 2006). A subsequent study has shown that condensin mediates the centromeric localization of Pol III genes, which is negatively correlated with Pol III transcription (Iwasaki et al. 2010). Remarkably, a mutation in TFIIIC suppresses the temperature sensitivity of a condensin mutant by restoring the centromere localization of Pol III genes. Thus, condensin-mediated clustering of Pol III genes at centromeres appears to counteract their transcription during interphase and also affects global condensation and segregation of chromosomes in mitosis.

\section{DNA damage response and repair}

Mitotic cell death (MCD) is a poorly characterized type of cell death that is triggered when extensive double-strand 
breaks (DSBs) are introduced into cells deficient in the p53-mediated G2/M checkpoint. In HeLa cells, for instance, MCD accompanies premature activation of Cdk1 and unscheduled condensation of chromatin into coalesced nucleoli, followed by the formation of micronuclei. Interestingly, it was found that condensin I, but not condensin II, is recruited to the condensing chromatin under this condition (Blank et al. 2006). This simple observation not only demonstrates that the MCD-associated chromatin condensation is mechanistically distinct from apoptosis-associated condensation, but also offers an experimental system for studying how condensins I and II might be differentially regulated. An independent study using HeLa cells reported that condensin I is involved in DNA single-strand break (SSB) repair, possibly through its interaction with poly(ADP-ribose) polymerase 1 (PARP-1) and the base excision repair factor XRCC1 (Heale et al. 2006). PARP-1 appears to contribute to the retention, but not the initial recruitment, of condensin I to DNA damage sites (Kong et al. 2011).

As mentioned earlier, a recent genetic study in $A$. thaliana has shown that condensin II is likely to be involved in DSB response and repair (Sakamoto et al. 2011). A genetic screen for mutants that exhibit hypersensitivity to excess boron identified two mutants (heb1-1 and heb2-1) that turned out to be defective in the genes encoding the CAP-G2 and CAP-H2 subunits of condensin II, respectively. The boron toxicity is most likely to involve incidence of DSBs, although the underlying mechanism remains to be clarified. This study also uncovered the somewhat surprising notion that condensin II is not essential for cell proliferation in this organism, thereby opening a way to dissect the functional involvement of condensin II in DSB response and repair in depth.

In $S$. pombe, a point mutation in the SMC2/Cut14 hinge domain confers defects in both DNA damage repair and mitotic chromosome condensation (Akai et al. 2011). Notably, a mutation in replication protein A (RPA), an ssDNA-binding protein, rescues both phenotypes, implying that condensin's DNA-reannealing activity might counteract RPA-mediated ssDNA coating (see further discussion below). The genetic properties of this mutation overlap with, but are not identical to, those of a mutation previously found in the CAP-H/Cnd2 subunit of $S$. pombe condensin (Aono et al. 2002), suggesting the existence of a complex regulatory network surrounding the interphase and mitotic functions of condensin.

\section{Meiotic chromosome function and segregation}

Not surprisingly, condensins also play crucial roles in meiotic chromosome architecture, dynamics, and functions. It is important to mention, however, that no meiosis-specific condensin subunits have been identified so far in any organisms. This situation contrasts with the cohesin fields, where the discovery and subsequent characterization of meiosis-specific subunits have greatly contributed to and accelerated our current understanding of the unique behaviors of chromosomes during meiosis (Petronczki et al. 2003).

\section{Mammals}

Currently, information regarding condensin functions in mammalian meiosis remains very limited. An early study using mouse spermatocytes reported that a condensin I subunit (CAP-H) is concentrated on a fuzzy axial structure within chromatids in both meiosis I and meiosis II (Viera et al. 2007). Whereas the existence of condensins I and II was initially reported in pig oocytes (Lisková et al. 2010), a more recent study in mouse oocytes has described a comprehensive picture of the dynamics and function of condensins I and II (Lee et al. 2011). At prophase of meiosis I (prophase I), condensin I is present in the cytoplasm, whereas condensin II is localized within the nucleus. After germinal vesicle breakdown, condensin II associates with chromosomes and is concentrated onto chromatid axes of bivalent chromosomes by metaphase I. In contrast, condensin I localizes primarily around centromeric regions at metaphase I and starts to associate with chromosome arms only after anaphase I. Together with results from antibody injection experiments, it has been proposed that during construction of bivalent chromosomes, condensin II plays a primary role in resolving sister chromatid axes, whereas condensin I may contribute to monopolar attachment of sister kinetochores by assembling a unique centromeric structure underneath (Lee et al. 2011). Future studies should address potential functions of condensins I and II at early stages of meiosis (e.g., pachytene), possibly combined with conditional knockout approaches.

\section{D. melanogaster}

The D. melanogaster genome encodes the orthologs of the CAP-D3 and CAP-H2 subunits of condensin II. Although mitotic cells express them, it appears that they do not play major functions in mitotic chromosome assembly or segregation (Savvidou et al. 2005). Instead, a recent genetic study has shown that both CAP-D3 and CAP-H2 are necessary for male fertility (Hartl et al. 2008b). Cap-H2 mutants display defects in chromosome territory formation in prophase I, which in turn result in a failure to resolve heterologous and/or homologous chromosomes in anaphase I. Thus, the putative condensin II function is required for not only segregating homologous chromosomes, but also individualizing heterologous chromosomes. Moreover, another study provided evidence that CAP-G, a condensin I subunit, is required for disassembly of the synaptonemal complex (SC) and keeping chromosomes in their metaphase I configuration in female meiosis (Resnick et al. 2008). Neither study has localized the condensin subunits to Drosophila meiotic chromosomes.

\section{C. elegans}

An early study had shown that RNAi-based depletion of SMC2/MIX-1 or SMC4/SMC-4 causes defects in chromosome segregation during meiosis II but not during meiosis I (Hagstrom et al. 2002). A subsequent genetic study performed under a tighter condition, however, demonstrated 
that the condensin II subunit CAP-D3/HCP6 is required for chromosome segregation in both meiosis I and meiosis II (Chan et al. 2004). Condensin II associates with sister chromatids during diplotene and diakinesis of prophase I and mediates their compaction and resolution, thereby contributing to formation of bivalent chromosomes. Unlike in mitosis, chromosomal loading of condensin I does not require aurora $B_{\text {; }}$ rather, the kinase regulates spatial distribution of condensin I by restricting it to the short arm of the bivalent (Collette et al. 2011).

The occurrence of crossover $(\mathrm{CO})$ recombination is essential for accurate chromosome segregation in meiosis. The distribution of $\mathrm{CO}$ is tightly regulated so that in C. elegans, a single $\mathrm{CO}$ forms between each homolog pair. Recent studies provided evidence that condensin I plays a major role in controlling $\mathrm{CO}$ distribution, whereas condensin II has a minor yet nonredundant function in this process (Tsai et al. 2008; Mets and Meyer 2009). Condensins could help limit the number of DSBs and COs through their activity to compact the meiotic chromosome axis. The localization of the two condensin complexes in pachytene chromosomes remains to be determined.

\section{S. cerevisiae}

The single condensin complex localizes to the axial core of pachytene chromosomes and contributes to their axial compaction in S. cerevisiae (Yu and Koshland 2003). Condensin mutants display pleiotropic defects in SC assembly, homolog pairing, and DSB processing. In these mutants, chromosome bridges are often observed during anaphase I and II, indicating that condensin function is required for proper segregation of chromosomes in both meiotic divisions. It was also reported that condensin helps recruit Polo/Cdc5 to meiotic chromosomes, which in turn contributes to cohesin release through its direct phosphorylation (Yu and Koshland 2005).

In $S$. cerevisiae, a four-subunit complex known as monopolin plays a central role in co-orienting sister kinetochores during meiosis I (Rabitsch et al. 2003; Corbett et al. 2010). A recent study suggested that condensin contributes to properly localizing a monopolin subunit (Mam1) at kinetochores, thereby ensuring co-orientation (Brito et al. 2010). Interestingly, two of the monopolin subunits, Csm1 and Lrs4, have an independent function during mitosis: recruiting condensin to the RFB within the rDNA repeat (Johzuka and Horiuchi 2009). Thus, condensin works closely with the monopolin subunits in both meiosis and mitosis, but does so in different contexts. In S. pombe, the orthologs of Csm1 and Lrs4 play a role in recruiting condensin to mitotic kinetochores, rather than contributing to meiotic co-orientation (Tada et al. 2011). It remains unknown whether metazoan species possess functional equivalents of Csm1 and Lrs4.

\section{A. thaliana}

As mentioned earlier, A. thaliana has two paralogous $S M C 2$ genes (AtCAP-E1 and AtCAP-E2) that have redundant functions in mitosis. Plants with a reduced dose of functional SMC2 (i.e., $E 1^{+/-} E 2^{-/-}$) display defects in chromosome segregation in meiosis I (Siddiqui et al. 2003), and the homozygous SMC4 mutation (AtCAP$\mathrm{C}^{-/-}$) causes a failure in gametogenesis in both females and males (Siddiqui et al. 2006). On the other hand, it appears that homozygous mutations in the genes encoding the CAP-G2 and CAP-H2 subunits of condensin II can produce viable gametes (Sakamoto et al. 2011). These results suggest that condensin I alone might be sufficient to support meiotic chromosome segregation in A. thaliana, a situation strikingly different from that in D. melanogaster or C. elegans.

\section{Cell type-specific functions and development}

Condensin subunits are widely expressed in proliferating cells. Recent studies, however, have started to provide evidence that a subtle disturbance of condensin functions often causes cell type- or tissue-specific defects, thereby unveiling the very rich biology beneath.

\section{Embryonic stem (ES) cells}

A recent RNAi screen for genes required for the viability of mouse ES cells has identified the Smc2 and Smc4 genes (Fazzio and Panning 2010). ES cells appear to be more sensitive to loss of condensin functions compared with somatic cells, and condensin-deficient ES cells display unique defective phenotypes that are not observed in somatic cells, such as massive enlargement of interphase nuclei and metaphase arrest. Moreover, some epigenetic modifications, such as $\mathrm{H} 3 \mathrm{~K} 9 \mathrm{me}, \mathrm{H} 3 \mathrm{~S} 10 \mathrm{ph}$, and $5 \mathrm{meC}$, are altered in condensin-depleted ES cells. It has become increasingly clear that the structural and functional features of chromatin in ES cells are strikingly different from those in somatic cell, possibly to maintain their developmental plasticity (Meshorer and Misteli 2006). ES cells are likely to provide an important and powerful system for studying the potential functions of condensins in epigenetic regulation and cell differentiation.

\section{T-cell development}

A forward genetic screen for recessive genes involved in T-cell development identified a mutant mouse strain, termed nessy, that turned out to carry a point mutation in the gene encoding the CAP-H2 (also known as kleisin $\beta$ ) subunit of condensin II (Gosling et al. 2007). The homozygous mutant mouse is viable and fertile and displays no apparent defect in B-cell development, precluding a general defect in chromosome condensation and segregation. Thus, this particular mutation causes a specific defect in T-cell development and a failure in normal immune response (Gosling et al. 2008) while preserving the essential function of condensin II. A more recent study has reported that nuclear chromatin rapidly condenses during thymocyte development in a condensin II-dependent manner and that this condensation is required for proper T-cell development and maintenance of the quiescent state (Rawlings et al. 2011). Upon T-cell activation, the chromatin decondenses again, making the transcriptional 
activator Stat5 accessible to its target promoters. These results suggest that condensin II regulates a developmentally programmed cycle of chromatin condensation and decondensation that has a great impact on T-cell differentiation. Intriguingly, similar if not identical chromatin condensation is observed during erythroid cell differentiation, and the condensin II subunit CAP-G2 is implicated in this process and accompanying transcriptional repression (Xu et al. 2006).

\section{Primary microcephaly}

Primary microcephaly is a neurodevelopmental disorder characterized by reduced brain size and mental retardation in humans (Thornton and Woods 2009). MCPH1 (also known as microcephalin) is one of the eight gene products known to be responsible for this disease. Intriguingly, cells from MCPH1 patients (but not from other MCPH patients) display abnormal chromosome dynamics, including premature chromosome condensation (PCC) in G2 phase (Trimborn et al. 2004). Although the PCC phenotype may be a secondary consequence of a compromised G2/M checkpoint observed in MCPH1deficient cells (Alderton et al. 2006; Tibelius et al. 2009), other lines of evidence suggest that MCPH1 may work very closely with condensin II (Trimborn et al. 2006; Wood et al. 2008). In fact, a more recent study has demonstrated that the $\mathrm{N}$-terminal domain of human MCPH1 (hMCPH1) is able to specifically inhibit the action of condensin II in Xenopus egg extracts and that its central domain has an auxiliary role in shaping metaphase chromosomes by physically interacting with condensin II (Yamashita et al. 2011). Despite these observations, it is rather surprising to find that $\mathrm{MCPH1}$-deficient cells segregate their ill-shaped chromosomes in a normal time frame. Mcph1 knockout mice are viable (Liang et al. 2010) and display microcephaly (Gruber et al. 2011). It is currently unknown whether the MCPH1's ability to regulate condensin II functions might be directly relevant to the etiology of microcephaly. The prevalent view in the field is that loss of MCPH gene functions causes an uncoupling between cell cycle progression and the centrosome cycle, which in turn compromises proliferation of neuroprogenitor cells with a highly unique division mode (Thornton and Woods 2009). Condensin II and MCPH1 could, in fact, function as components of the signaling network that regulates mitotic entry in response to a range of cellular stresses (Hirano 2005; Chin and Yeong 2010). Whatever the mechanism might be, it is important to emphasize that MCPH1 microcephaly represents the first example of human diseases that accompany misregulation of the chromosome condensation machinery.

\section{Cancers and tissue growth}

Genome instability is a hallmark of cancers. Given the critical role of condensins in proper assembly and segregation of chromosomes, it is formally possible that subtle perturbations of their functions might promote genome instability, eventually contributing to tumorigenesis. Although no direct evidence for this idea has been reported so far, potentially interesting observations can be found in the literature. For instance, somatic point mutations in the SMC2/CAP-E and SMC4/CAP-C subunits have been identified in several cases of pyothoraxassociated lymphoma in humans (Ham et al. 2007). In zebrafish, a mutation in the condensin I CAP-G subunit increases genomic imbalances and the rate of apoptosis in the retina (Seipold et al. 2009). In Drosophila, it has been reported that a retinoblastoma family protein (Rfb1) is required for efficient localization of condensin II with chromatin by physically interacting with its subunit, CAP-D3 (Longworth et al. 2008). Finally, a mutation in the condensin I CAP-G subunit has been found to be tightly associated with an increase in body size at puberty in cattle (Setoguchi et al. 2011). The corresponding syntenic region in human chromosome 4 is also known be to be associated with adult height, raising the possibility that a condensin I variant might affect skeletal growth in mammals.

\section{Molecular mechanisms of action}

Despite the rapid accumulation of information regarding the biology of condensins, our understanding of their molecular mechanisms of action remains poor. The large size and intricate subunit architecture of the complexes have made it a big challenge to reconstitute active complexes from recombinant subunits (Onn et al. 2007). Nonetheless, extensive efforts using a wide variety of approaches are now gathering valuable information about the structure and mechanics of condensins. For reviews on the mechanistic aspects of condensins, see also Swedlow and Hirano (2003), Hirano (2006), Cuylen and Haering (2011), and Baxter and Aragón (2012).

\section{Ensuring dsDNA: a preparatory step for subsequent coiling?}

One of the activities that have been found to be associated with condensins is a DNA-reannealing activity that promotes conversion of complementary ssDNAs into dsDNA (Fig. 4A, panel a). This activity does not require a five-subunit holocomplex: The SMC2-SMC4 dimer is sufficient to support DNA reannealing in the absence of ATP (Sakai et al. 2003). Remarkably, the SMC dimer is able to reanneal ssDNAs precoated with RPA, leaving the final products of dsDNA free of RPA and SMC (Akai et al. 2011). Several lines of evidence further indicate that the hinge domain of SMC subunits plays an important role in interacting with ssDNA (Hirano and Hirano 2006; Griese et al. 2010; Akai et al. 2011).

How, then, might this activity be physiologically relevant? It is reasonable to assume that ssDNA regions would not act as proper substrates for the formation of mitotic chromosomes and must therefore be "fixed" before initiating such processes. The postulated fixation step would involve not only rewinding of ssDNA regions but also removal of ssDNA-binding proteins or RNAs that would otherwise block dsDNA formation (Yanagida 2009). Thus, condensins' reannealing activity might help 
A

$\mathbf{B}$

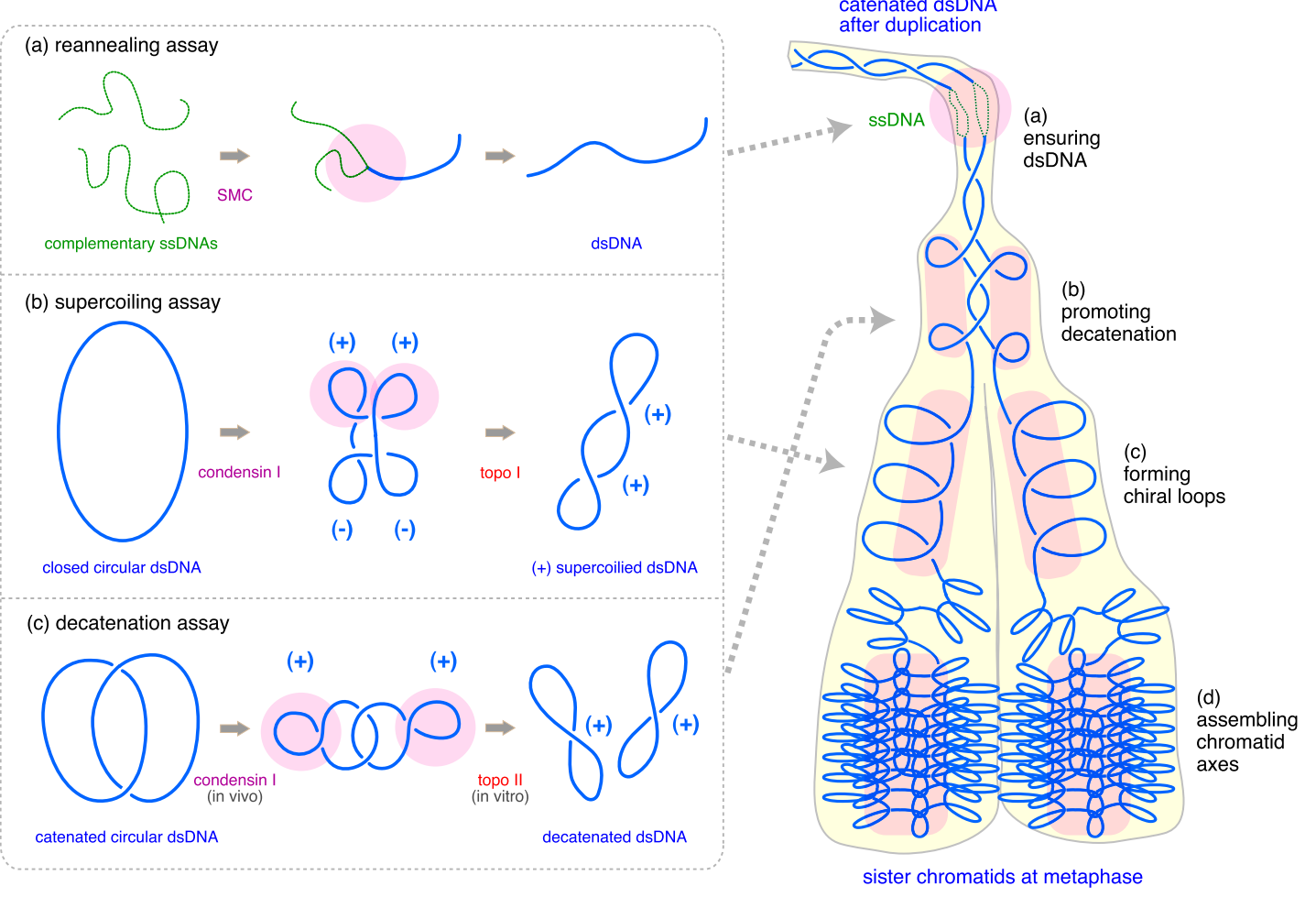

Figure 4. Molecular mechanisms of action of eukaryotic condensins. (A, panel $a$ ) Reannealing assay. The SMC2-SMC4 dimer is able to convert complementary ssDNAs (green dotted line) into dsDNA (blue solid line) in vitro. This reaction does not require ATP binding or hydrolysis. (Panel $b$ ) Supercoiling assay. Condensin I introduces positive supercoils into closed circular DNA in the presence of topo I. This reaction requires ATP binding and hydrolysis by the SMC subunits. (Panel $c$ ) Decatenation assay. Condensin's action allows the accumulation of positive supercoils into catenated circular DNAs in topo II-depleted, mitotically arrested cells. When these templates are purified and incubated with topo II in vitro, they are quickly decatenated before being relaxed. $(B)$ An integrated model of how condensins might promote conformational changes of chromosomes. (Step a) The reannealing activity helps ensure dsDNA, a prerequisite for subsequent coiling steps. (Step $b$ ) Introduction of positive superhelical tension into catenated sister DNAs promotes topo II-mediated decatenation, thereby facilitating the resolution of sister chromatids. (Step $c$ ) Continued positive supercoiling of the decatenated (and therefore liberated) sister DNAs helps the formation of chiral loops. (Step $d$ ) Oligomerization or higher-order assembly of condensins might further facilitate and stabilize ordered folding of chromatin fibers, eventually leading to the assembly of sister chromatid axes. It is most likely that the four steps depicted here are not completely separable from each other and must occur in a spatiotemporally coordinated fashion.

ensure that dsDNA is ready for subsequent reactions (Fig. $4 \mathrm{~B}$, step a). It is tempting to speculate that condensins use a similar basic mechanism to regulate other interphase functions, such as fine-tuning of transcription (e.g., dosage compensation) and suppression of undesired recombination (e.g., rDNA copy number maintenance). Moreover, it is important to note that the reannealing activity bears a mechanistic parallel to the positive supercoiling activity discussed below: The former involves rewinding of ssDNA into dsDNA, whereas the latter accompanies overwinding of dsDNA.

\section{Supercoiling and decatenating dsDNA}

Perhaps the best-characterized activity of condensin I is its ability to introduce positive superhelical tension into dsDNA, which can be assayed in vitro by using closed circular DNA as a template in the presence of type I topoisomerases (Fig. 4A, panel b). Unlike the reannealing activity, this positive supercoiling activity demands the five-subunit holocomplex and ATP hydrolysis by the SMC subunits (Kimura and Hirano 2000). It has been detected widely in many eukaryotic species, including humans (Kimura et al. 2001), X. laevis (Kimura and Hirano 1997), C. elegans (Hagstrom et al. 2002), and $S$. cerevisiae (St-Pierre et al. 2009). Moreover, as judged by a modified assay, condensin I is able to organize multiple supercoils into an ordered, solenoidal form (Kimura et al. 1999). It has been proposed that the positive supercoiling activity could promote ordered folding of chromatin fibers to initiate the formation of chiral (positively supercoiled) loops in eukaryotes (Fig. 4B, step c; Swedlow and Hirano 2003; Hirano 2006).

A recent study in $S$. cerevisiae has provided evidence that condensin is indeed responsible for accumulation of positive supercoils on catenated sister DNAs in vivo under the condition in which topo II is depleted (Baxter et al. 2011). Remarkably, when tested in vitro, catenated plas- 
mids with positive supercoils are more susceptible to topo II-mediated decatenation than those with no supercoils (Fig. 4A, panel c), suggesting that condensin-mediated positive supercoiling could facilitate decatenation of sister DNA molecules during mitosis (Fig. 4B, step b). It is reasonable to speculate that when this action of condensin is compromised, catenanes between sister DNAs fail to be removed completely, thereby causing defects in sister chromatid resolution and segregation in anaphase (e.g., Coelho et al. 2003; Hudson et al. 2003; D'Ambrosio et al. 2008a). In the future, it will be important to address how positive superhelical tension imposed by condensin might alter nucleosome structures (Bancaud et al. 2007) and how topo II might act on such nucleosome templates (Roca 2009).

A pair of recent studies has shown that E. coli MukB physically interacts with ParC, the DNA-binding subunit of a type II topoisomerase (topo IV), and stimulates its activity in vitro (Hayama and Marians 2010; Li et al. 2010). Although these observations are highly illuminating, careful consideration will be required before integrating all data currently available because there exist some mechanistic differences in the eukaryotic and prokaryotic decatenation systems (Hirano 2000; Postow et al. 2001).

\section{How might condensins work?}

The mechanism by which the condensin complexes might interact with DNA to induce its conformational changes is unknown. A recent study using minichromosomes isolated from yeast cells has provided evidence that condensin encircles dsDNA, thereby forming topological links_a mechanism similar to that proposed for cohesin-DNA interactions (Cuylen et al. 2011). It is attractive to speculate that whereas cohesin holds two different sister DNA strands together, a single condensin complex might link two distant segments of a sister chromatid to promote its folding and compaction. Indeed, this idea is not incompatible with the chiral looping model of condensin's action, although condensin-DNA interactions are clearly more complex than those predicted from a simple topological entrapment (Stray and Lindsley 2003; Stray et al. 2005; Hudson et al. 2008).

Some of the previous models predicted that condensins could oligomerize or form a higher-order assembly to fold a chromatin fiber during the process of chromosome condensation (Swedlow and Hirano 2003; Hirano 2006; Graumann and Knust 2009). In fact, positive supercoiling of DNA is likely to be a net product of reactions involving multiple condensin I complexes, and a single DNA molecule manipulation assay using magnetic tweezers also provides strong support for cooperative actions of condensin I (Strick et al. 2004). It is possible that highly dynamic condensin-DNA interactions are further modified or stabilized by condensin-condensin interactions, which in turn contribute to assembling sister chromatid axes (Fig. 4B, step d; Maeshima and Laemmli 2003; Ono et al. 2003; Kireeva et al. 2004). Moreover, the idea of oligomerization gains support from several different experiments using bacterial condensins, including electron microscopy (Matoba et al. 2005; Fuentes-Perez et al.
2012), a single DNA molecule assay (Cui et al. 2008), and a combination of structural and biochemical analyses (Woo et al. 2009). Particularly important is the proposal that ATP-dependent engagement of MukB head domains disrupts a MukB-MukF interaction, thereby providing an opportunity to initiate oligomerization of multiple MukBEF complexes (Woo et al. 2009). Future studies using the eukaryotic and prokaryotic complexes should clarify exactly how the ATP-binding and hydrolysis cycle of their SMC core subunits might be coupled with condensin-DNA interactions as well as putative condensincondensin interactions.

\section{Conclusions and perspective}

Although the condensin complexes were originally discovered as central players in mitotic chromosome assembly and segregation, recent studies revealed that they participate in an amazingly diverse array of chromosomal events. Evidence is also accumulating that condensins I and II fulfill nonoverlapping functions, behave differentially during the cell cycle, and are under the control of distinct sets of specific regulators. From an evolutionary point of view, the principal position of condensin I in mitotic segregation is conserved among virtually all eukaryotes. In contrast, condensin II is apparently used in different contexts among different species. Although these conceptual advances provide a valuable guide for future studies in the field, a number of outstanding questions remain to be answered. For example, the molecular mechanisms of action of condensins must be investigated and understood in depth. Because most of our current knowledge comes from analyses of condensin I, it will be of great importance to critically address to what extent the two condensin complexes might differentiate from each other at a mechanistic level. Moreover, it will be of great interest to explore cell type- and tissue-specific functions of condensins and their relevance to the development of organisms. Along these lines, functional cross-talk of condensins with epigenetic control will be an exciting area in future studies. The integration of knowledge from multidisciplinary approaches should eventually shed light on the evolutionary questions of how a primitive form of life might have devised a strategy for handling the long molecules of DNA and how such an early principle of genome organization might have evolved to help create the astonishing diversity of life that we see today on Earth.

\section{Acknowledgments}

I thank members of the Hirano laboratory for critically reading the manuscript. I am also grateful to many colleagues in the condensin field for stimulating discussions. The work done in my laboratory was supported by Grant-in-Aid for Specially Promoted Research (20002010).

\section{References}

Abe S, Nagasaka K, Hirayama Y, Kozuka-Hata H, Oyama M, Aoyagi Y, Obuse C, Hirota T. 2011. The initial phase of 
chromosome condensation requires Cdk1-mediated phosphorylation of the CAP-D3 subunit of condensin II. Genes Dev 25: 863-874.

Akai Y, Kurokawa Y, Nakazawa N, Tonami-Murakami Y, Suzuki Y, Yoshimura S-H, Iwasaki H, Shiroiwa Y, Nakamura $\mathrm{T}$, Shibata E, et al. 2011. Opposing role of condensin hinge against replication protein $\mathrm{A}$ in mitosis and interphase through promoting DNA annealing. Open Biol 1: 110023. doi: 10.1098/ rsob.110023.

Alderton GK, Galbiati L, Griffith E, Surinya KH, Neitzel H, Jackson AP, Jeggo PA, O'Driscoll M. 2006. Regulation of mitotic entry by microcephalin and its overlap with ATP signalling. Nat Cell Biol 8: 725-733.

Anderson DE, Losada A, Erickson HP, Hirano T. 2002. Condensin and cohesin display different arm conformations with characteristic hinge angles. J Cell Biol 156: 419-424.

Aono N, Sutani T, Tomonaga T, Mochida S, Yanagida M. 2002. $\mathrm{Cnd} 2$ has dual roles in mitotic condensation and interphase. Nature 417: 197-202.

Bancaud A, Wagner G, Conde E, Silva N, Lavelle C, Wong H, Mozziconacci J, Barbi M, Sivolob A, Le Cam E, et al. 2007. Nucleosome chiral transition under positive torsional stress in single chromatin fibers. Mol Cell 27: 135-147.

Baxter J, Aragón L. 2012. A model for chromosome condensation based on the interplay between condensin and topoisomerase II. Trends Genet 28: 110-117.

Baxter J, Sen N, Martínez VL, De Carandini MEM, Schvartzman JB, Diffley JFX, Aragón L. 2011. Positive supercoiling of mitotic DNA drives decatenation by topoisomerase II in eukaryotes. Science 331: 1328-1332.

Bazile F, St-Pierre J, D'Amours D. 2010. Three-step model for condensin activation during mitotic chromosome condensation. Cell Cycle 9: 3243-3255.

Belmont AS. 2006. Mitotic chromosome structure and condensation. Curr Opin Cell Biol 18: 1-7.

Bernad R, Sánchez P, Rivera T, Rodríguez-Corsino M, Boyarchuk E, Vassias I, Ray-Gallet D, Arnaoutov A, Dasso M, Almouzni G, et al. 2011. Xenopus HJURP and condensin II are required for CENP-A assembly. J Cell Biol 192: 569-582.

Bhat MA, Philp AV, Glover DM, Bellen HJ. 1996. Chromatid segregation at anaphase requires the barren product, a novel chromosome associated protein that interacts with topoisomerase II. Cell 87: 1103-1114.

Blank M, Lerenthal Y, Mittelman L, Shiloh Y. 2006. Condensin I recruitment and uneven chromatin condensation precede mitotic cell death in response to DNA damage. J Cell Biol 174: 195-206.

Blauwkamp TA, Csankovszki G. 2009. Two classes of dosage compensation complex binding elements along Caenorhabditis elegans X chromosomes. Mol Cell Biol 29: 2023-2031.

Brito IL, Yu H-G, Amon A. 2010. Condensins promote coorientation of sister chromatids during meiosis I in budding yeast. Genetics 185: 55-64.

Cervantes MD, Coyne RS, Xi X, Yao M-C. 2006. The condensin complex is essential for amitotic segregation of bulk chromosomes, but not nucleoli, in the ciliate Tetrahymena thermophila. Mol Cell Biol 26: 4690-4700.

Chan RC, Severson AF, Meyer BJ. 2004. Condensin restructures chromosomes in preparation for meiotic divisions. J Cell Biol 167: 613-625.

Chin CF, Yeong FM. 2010. Safeguarding entry into mitosis: The antephase checkpoint. Mol Cell Biol 30: 22-32.

Clemente-Blanco A, Mayán-Santos M, Schneider D, Machín F, Jarmuz A, Tschochner H, Aragón L. 2009. Cdc14 inhibits transcription by RNA polymerase I during anaphase. Nature 458: 219-222.
Coelho P, Queiroz-Mechado J, Sunkel CE. 2003. Condensindependent localisation of topoisomerase II to an axial chromosomal structure is required for sister chromatid resolution during mitosis. J Cell Sci 116: 4763-4776.

Collette KS, Petty EL, Golenberg N, Bembenek JN, Csankovszki G. 2011. Different roles for Aurora B in condensin targeting during mitosis and meiosis. J Cell Sci 124: 3684-3694.

Corbett KD, Yip CK, Ee L-S, Walz T, Amon A, Harrison SC. 2010. The monopolin complex crosslinks kinetochore components to regulate chromosome-microtubule attachments. Cell 142: 556-567.

Csankovszki G, Collette K, Spahl K, Carey J, Snyder M, Petty E, Patel U, Tabuchi T, Liu H, McLeod I, et al. 2009a. Three distinct condensin complexes control C. elegans chromosome dynamics. Curr Biol 19: 9-19.

Csankovszki G, Petty EL, Collette KS. 2009b. The worm solution: A chromosome-full of condensin helps gene expression go down. Chromosome Res 17: 621-635.

Cui Y, Petrushenko ZM, Rybenkov VV. 2008. MukB acts as a macromolecular clamp in DNA condensation. Nat Struct Mol Biol 15: 411-418.

Cuylen S, Haering CH. 2011. Deciphering condensin action during chromosome segregation. Trends Cell Biol 21: 552559.

Cuylen S, Metz J, Haering CH. 2011. Condensin structures chromosomal DNA through topological links. Nat Struct Mol Biol 18: 894-901.

D'Ambrosio C, Kelly G, Shirahige K, Uhlmann F. 2008a. Condensin-dependent rDNA decatenation introduces a temporal pattern to chromosome segregation. Curr Biol 18: 10841089.

D'Ambrosio C, Schmidt CK, Katou Y, Kelly G, Itoh T, Shirahige K, Uhlmann F. 2008b. Identification of cis-acting sites for condensin loading onto budding yeast chromosomes. Genes Dev 22: 2215-2227.

Dej KJ, Ahn C, Orr-Weaver TL. 2004. Mutations in the Drosophila condensin subunit dCAP-G: Defining the role of condensin for chromosome condensation in mitosis and gene expression in interphase. Genetics 168: 895-906.

Eisen JA, Coyne RS, Wu M, Wu D, Thiagarajan M, Wortman JR, Badger JH, Ren Q, Amedeo P, Jones KM, et al. 2006. Macronuclear genome sequence of the ciliate Tetrahymena thermophila, a model eukaryote. PLoS Biol 4: e286. doi: 10.1371/ journal.pbio.0040286.

Ercan S, Lieb JD. 2009. C. elegans dosage compensation: A window into mechanisms of domain-scale gene regulation. Chromosome Res 17: 215-227.

Ercan S, Giresi PG, Whittle CM, Zhang X, Green RD, Lieb JD. 2007. X chromosome repression by localization of the C. elegans dosage compensation machinery to sites of transcription initiation. Nat Genet 39: 403-408.

Ercan S, Dick L, Lieb J. 2009. The C. elegans dosage compensation complex propagates dynamically and independently of X chromosome sequence. Curr Biol 19: 1777-1787.

Fazzio TG, Panning B. 2010. Condensin complexes regulate mitotic progression and interphase chromatin structure in embryonic stem cells. J Cell Biol 188: 491-503.

Fennell-Fezzie R, Gradia SD, Akey D, Berger JM. 2005. The MukF subunit of Escherichia coli condensin: Architecture and functional relationship to kleisins. EMBO J 24: 19211930.

Freeman L, Aragon-Alcaide L, Strunnikov AV. 2000. The condensin complex governs chromosome condensation and mitotic transmission of rDNA. I Cell Biol 149: 811-824.

Fuentes-Perez ME, Gwynn EJ, Dillingham MS, Moreno-Herrero F. 2012. Using DNA as a fiducial marker to study SMC 
complex interactions with the atomic force microscope. Biophys J 102: 839-848.

Gasser SM, Laemmli UK. 1987. Improved methods for the isolation of individual and clustered mitotic chromosomes. Exp Cell Res 173: 85-98.

Gerlich D, Hirota T, Koch B, Peters J-M, Ellenberg J. 2006. Condensin I stabilizes chromosomes mechanically through a dynamic interaction in living cells. Curr Biol 16: 333-344.

Giet R, Glover DM. 2001. Drosophila Aurora B kinase is required for histone $\mathrm{H} 3$ phosphorylation and condensin recruitment during chromosome condensation and to organize the central spindle during cytokinesis. J Cell Biol 152: 669682.

Gloyd M, Ghirlando R, Matthews LA, Guarné A. 2007. MukE and MukF form two distinct high affinity complexes. J Biol Chem 282: 14373-14378.

Gosling KM, Makaroff LE, Theodoratos A, Kim Y-H, Whittle B, Rui L, Wu H, Hong NA, Kennedy GC, Fritz J-A, et al. 2007. A mutation in a chromosome condensin II subunit, kleisin b, specifically disrupts T cell development. Proc Natl Acad Sci 104: $12445-12450$.

Gosling KM, Goodnow CC, Verma NK, Fahrer AM. 2008. Defective T-cell function leading to reduced antibody production in a kleisin- $\beta$ mutant mouse. Immunology 125: 208217.

Graumann PL, Knust T. 2009. Dynamics of the bacterial SMC complex and SMC-like proteins involved in DNA repair. Chromosome Res 17: 265-275.

Green LC, Kalitsis P, Chang TM, Cipetic M, Kim JH, Marshall O, Turnbull L, Whitchurch CB, Vagnarelli P, Samejima K, et al. 2012. Contrasting roles of condensin I and II in mitotic chromosome formation. J Cell Sci 125: 1591-1604.

Griese JJ, Witte G, Hopfner K-P. 2010. Structure and DNA binding activity of the mouse condensin hinge domain highlight common and diverse features of SMC proteins. Nucleic Acids Res 38: 3454-3465.

Gruber S, Errington J. 2009. Recruitment of condensin to replication origin regions by $\mathrm{ParB} / \mathrm{SpoOJ}$ promotes chromosome segregation in B. subtilis. Cell 137: 685-696.

Gruber R, Zhou Z, Sukchev M, Joerss T, Frappart P-O, Wang Z-Q. 2011. MCPH1 regulates the neuroprogenitor division mode by coupling the centrosomal cycle with mitotic entry through the Chk1-Cdc25 pathway. Nat Cell Biol 13: 13251334.

Haeusler RA, Pratt-Hyatt M, Good PD, Gipson TA, Engelke DR. 2008. Clustering of yeast tRNA genes is mediated by specific association of condensin with tRNA gene transcription complexes. Genes Dev 22: 2204-2214.

Hagstrom KA, Holmes VF, Cozzarelli NR, Meyer BJ. 2002. C. elegans condensin promotes mitotic chromosome architecture, centromere organization, and sister chromatid segregation during mitosis and meiosis. Genes Dev 16: 729742.

Ham MF, Takakuwa T, Rahadiani N, Tresnasari K, Nakajima H, Aozasa K. 2007. Condensin mutations and abnormal chromosomal structures in pyothorax-assocaited lymphoma. Cancer Sci 98: 1041-1047.

Hartl TA, Smith HF, Bosco G. 2008a. Chromosome alignment and transvection are antagonized by condensin II. Science 322: $1384-1387$.

Hartl TA, Sweeney SJ, Knepler PJ, Bosco G. 2008b. Condensin II resolves chromosomal associations to enable anaphase I segregation in Drosophila male meiosis. PLoS Genet 4: e1000228. doi: 10.1371/journal.pgen.1000228.

Hayama R, Marians KJ. 2010. Physical and functional interaction between the condensin MukB and the decatenase topo- isomerase IV in Escherichia coli. Proc Natl Acad Sci 107: $18826-18831$.

Heale JT, Ball AR, Schmiesing JA, Kim J-S, Kong X, Zhou S, Hudson DF, Earnshaw WC, Yokomori K. 2006. Condensin I interacts with the PARP-1-XRCC1 complex and functions in DNA single-strand break repair. Mol Cell 21: 837-848.

Hegemann B, Hutchins JRA, Hudecz O, Novatchkova M, Rameseder J, Sykora MM, Liu S, Mazanek M, Lénárt P, Hériché J-K, et al. 2011. Systematic phosphorylation analysis of human mitotic protein complexes. Sci Signal 4: rs12. doi: 10.1126/scisignal.2001993.

Hiraga S. 2000. Dynamic localization of bacterial and plasmid chromosomes. Annu Rev Genet 34: 21-59.

Hirano T. 2000. Chromosome cohesion, condensation and separation. Annu Rev Biochem 69: 115-144.

Hirano T. 2005. Condensins: Organizing and segregating the genome. Curr Biol 15: R265-R275. doi: 10.1016/j.cub. 2005.03.037.

Hirano T. 2006. At the heart of the chromosome: SMC proteins in action. Nat Rev Mol Cell Biol 7: 311-322.

Hirano M, Hirano T. 2004. Positive and negative regulation of SMC-DNA interactions by ATP and accessory proteins. EMBO J 23: 2664-2673.

Hirano M, Hirano T. 2006. Opening closed arms: Long-distance activation of SMC ATPase by hinge-DNA interaction. Mol Cell 21: 175-186.

Hirano T, Mitchison TJ. 1994. A heterodimeric coiled-coil protein required for mitotic chromosome condensation in vitro. Cell 79: 449-458.

Hirano T, Kobayashi R, Hirano M. 1997. Condensins, chromosome condensation protein complexes containing XCAP-C, XCAP-E and a Xenopus homolog of the Drosophila Barren protein. Cell 89: 511-521.

Hirota T, Gerlich D, Koch B, Ellenberg J, Peters JM. 2004. Distinct functions of condensin I and II in mitotic chromosome assembly. J Cell Sci 117: 6435-6445.

Hudson DF, Vagnarelli P, Gassmann R, Earnshaw WC. 2003. Condensin is required for nonhistone protein assembly and structural integrity of vertebrate chromosomes. Dev Cell 5: 323-336.

Hudson DF, Ohta S, Freisinger T, Macisaac F, Sennels L, Alves F, Lai F, Kerr A, Rappsilber J, Earnshaw W. 2008. Molecular and genetic analysis of condensin function in vertebrate cells. Mol Biol Cell 19: 3070-3079.

Hudson DF, Marshall KM, Earnshaw WC. 2009. Condensin: Architect of mitotic chromosomes. Chromosome Res 17: 131-144.

Ide S, Miyazaki T, Maki H, Kobayashi T. 2010. Abundance of ribosomal RNA gene copies maintains genome integrity. Science 327: 693-696.

Iwasaki O, Tanaka A, Tanizawa H, Grewal S, Noma K. 2010. Centromeric localization of dispersed pol III genes in fission yeast. Mol Biol Cell 21: 254-265.

Jans J, Gladden JM, Ralston EJ, Pickle CS, Michel AH, Pferdehirt RR, Eisen MB, Meyer BJ. 2009. A condensin-like dosage compensation complex acts at a distance to control expression throughout the genome. Genes Dev 23: 602-618.

Johzuka K, Horiuchi T. 2007. RNA polymerase I transcription obstructs condensin association with 35S rRNA coding regions and can cause contraction of long repeat in Saccharomyces cerevisiae. Genes Cells 12: 759-771.

Johzuka K, Horiuchi T. 2009. The cis element and factors required for condensin recruitment to chromosomes. Mol Cell 34: 26-35.

Johzuka K, Terasawa M, Ogawa H, Ogawa T, Horiuchi T. 2006. Condensin loaded onto the replication fork barrier site in the 
rRNA gene repeats during $S$ phase in a FOB1-dependent fashion to prevent contraction of a long repetitive array in Saccharomyces cerevisiae. Mol Cell Biol 26: 2226-2236.

Kawamura R, Pope LH, Christensen MO, Sun M, Terekhova K, Boege F, Mielke C, Andersen AH, Marko JF. 2010. Mitotic chromosomes are constrained by topoisomerase II-sensitive DNA entanglements. J Cell Biol 188: 653-663.

Kimura K, Hirano T. 1997. ATP-dependent positive supercoiling of DNA by $13 \mathrm{~S}$ condensin: A biochemical implication for chromosome condensation. Cell 90: 625-634.

Kimura K, Hirano T. 2000. Dual roles of the 11S regulatory subcomplex in condensin functions. Proc Natl Acad Sci 97: 11972-11977.

Kimura K, Hirano M, Kobayashi R, Hirano T. 1998. Phosphorylation and activation of $13 \mathrm{~S}$ condensin by Cdc2 in vitro. Science 282: 487-490.

Kimura K, Rybenkov VV, Crisona NJ, Hirano T, Cozzarelli NR. 1999. 13S condensin actively reconfigures DNA by introducing global positive writhe: Implications for chromosome condensation. Cell 98: 239-248.

Kimura K, Cuvier O, Hirano T. 2001. Chromosome condensation by a human condensin complex in Xenopus egg extracts. I Biol Chem 276: 5417-5420.

Kireeva N, Lakonishok M, Kireev I, Hirano T, Belmont AS. 2004. Visualization of early chromosome condensation: A hierarchical folding, axial glue model of chromosome structure. I Cell Biol 166: 775-785.

Kong X, Stephens J, Ball AR, Heale JT, Newkirk DA, Berns MW, Yokomori K. 2011. Condensin I recruitment to base damageenriched DNA lesions is modulated by PARP1. PLOS ONE 6: e23548. doi: 10.1371/journal.pone.0023548.

Koonin EV. 2010. Preview. The incredible expanding ancestor of eukaryotes. Cell 140: 606-608.

Lai S-K, Wong C-H, Lee Y-P, Li H-Y. 2010. Caspase-3-mediated degradation of condensin Cap-H regulates mitotic cell death. Cell Death Differ 18: 996-1004.

Lavoie BD, Hogan E, Koshland D. 2004. In vivo requirements for rDNA chromosome condensation reveal two cell-cycle-regulated pathways for mitotic chromosome folding. Genes Dev 18: $76-87$.

Lee J, Ogushi S, Saitou M, Hirano T. 2011. Condensins I and II are essential for construction of bivalent chromosomes in mouse oocytes. Mol Biol Cell 22: 3465-3477.

Li Y, Stewart NK, Berger AJ, Vos S, Schoeffler AJ, Berger JM, Chait BT, Oakley MG. 2010. Escherichia coli condensin MukB stimulates topoisomerase IV activity by a direct physical interaction. Proc Natl Acad Sci 107: 18832-18837.

Liang Y, Gao H, Lin S-Y, Peng G, Huang X, Zhang P, Goss JA, Brunicardi FC, Multani AS, Chang S, et al. 2010. BRIT1/ $\mathrm{MCPH1}$ is essential for mitotic and meiotic recombination DNA repair and maintaining genomic stability in mice. PLoS Genet 6: e1000826. doi: 10.1371/journal.pgen.1000826.

Lipp JJ, Hirota T, Poser I, Peters J-M. 2007. Aurora B controls the association of condensin I but not condensin II with mitotic chromosomes. J Cell Sci 120: 1245-1255.

Lisková L, Susor A, Pivonková K, Sasková A, Karabínová P, Kubelka M. 2010. Detection of condensin I and II in maturing pig oocytes. Reprod Fertil Dev 22: 644-652.

Liu W, Tanasa B, Tyurina OV, Zhou TY, Gassmann R, Liu WT, Ohgi KA, Benner C, Garcia-Bassets I, Aggarwal AK, et al. 2010. PHF8 mediates histone H4 lysine 20 demethylation events involved in cell cycle progression. Nature 466: 508 512.

Longworth MS, Herr A, Ji JY, Dyson NJ. 2008. RBF1 promotes chromatin condensation through a conserved interaction with the Condensin II protein dCAP-D3. Genes Dev 22: 1011-1024.
MacCallum DE, Losada A, Kobayashi R, Hirano T. 2002. ISWI remodeling complexes in Xenopus egg extracts: Identification as major chromosomal components that are regulated by INCENP-aurora B. Mol Biol Cell 13: 25-39.

Maeshima K, Laemmli UK. 2003. A two-step scaffolding model for mitotic chromosome assembly. Dev Cell 4: 467-480.

Marko JF. 2008. Micromechanical studies of mitotic chromosomes. Chromosome Res 16: 469-497.

Matoba K, Yamazoe M, Mayanagi K, Morikawa K, Hiraga S. 2005. Comparison of MukB homodimer versus MukBEF complex molecular architectures by electron microscopy reveals a higher-order multimerization. Biochem Biophys Res Commun 333: 694-702.

McDonel P, Jans J, Peterson BK, Meyer BJ. 2006. Clustered DNA motifs mark X chromosomes for repression by a dosage compensation complex. Nature 444: 614-618.

Meshorer E, Misteli T. 2006. Chromatin in pluripotent embryonic stem cells and differentiation. Nat Rev Mol Cell Biol 7: 540-546.

Mets D, Meyer B. 2009. Condensins regulate meiotic DNA break distribution, thus crossover frequency, by controlling chromosome structure. Cell 139: 73-86.

Mora-Bermudez F, Gerlich D, Ellenberg J. 2007. Maximal chromosome compaction occurs by axial shortening in anaphase and depends on Aurora kinase. Nat Cell Biol 9: 822831.

Nakazawa N, Nakamura T, Kokubu A, Ebe M, Nagao K, Yanagida M. 2008. Dissection of the essential steps for condensin accumulation at kinetochores and rDNAs during fission yeast mitosis. J Cell Biol 180: 1115-1131.

Nakazawa N, Mehrotra R, Ebe M, Yanagida M. 2011. Condensin phosphorylated by the Aurora-B-like kinase Ark1 is continuously required until telophase in a mode distinct from Top2. J Cell Sci 124: 1795-1807.

Neuwald AF, Hirano T. 2000. HEAT repeats associated with condensins, cohesins and other chromosome-related complexes. Genome Res 10: 1445-1452.

Nishino Y, Eltsov M, Joti Y, Ito K, Takata H, Takahashi Y, Hihara S, Frangakis AS, Imamoto N, Ishikawa T, et al. 2012. Human mitotic chromosomes consist predominantly of irregularly folded nucleosome fibres without a $30-\mathrm{nm}$ chromatin structure. EMBO J 31: 1644-1653.

Noma K, Cam HP, Marata RJ, Grewal SI. 2006. A role for TFIIIC transcription factor complex in genome organization. Cell 125: 859-872.

Nousiainen M, Silljé HHW, Sauer G, Nigg EA, Körner R. 2006. Phosphoproteome analysis of the human mitotic spindle. Proc Natl Acad Sci 103: 5391-5396.

Oliveira RA, Coelho PA, Sunkel CE. 2005. The condensin I subunit Barren/CAP-H is essential for the structural integrity of centromeric heterochromatin during mitosis. Mol Cell Biol 25: 8971-8984.

Oliveira RA, Heidmann S, Sunkel CE. 2007. Condensin I binds chromatin early in prophase and displays a highly dynamic association with Drosophila mitotic chromosomes. Chromosoma 116: 259-274.

Onn I, Aono N, Hirano M, Hirano T. 2007. Reconstitution and subunit geometry of human condensin complexes. EMBO I 26: $1024-1034$

Ono T, Losada A, Hirano M, Myers MP, Neuwald AF, Hirano T. 2003. Differential contributions of condensin I and condensin II to mitotic chromosome architecture in vertebrate cells. Cell 115: 109-121.

Ono T, Fang Y, Spector D, Hirano T. 2004. Spatial and temporal regulation of condensins I and II in mitotic chromosome assembly in human cells. Mol Biol Cell 15: 3296-3308. 
Pagliuca FW, Collins MO, Lichawska A, Zegerman P, Choudhary JS, Pines J. 2011. Quantitative proteomics reveals the basis for the biochemical specificity of the cell-cycle machinery. Mol Cell 43: 406-417.

Petronczki M, Siomos MF, Nasmyth K. 2003. Un ménage à quatre: The molecular biology of chromosome segregation in meiosis. Cell 112: 423-440.

Petty EL, Collette KS, Cohen AJ, Snyder MJ, Csankovszki G. 2009. Restricting dosage compensation complex binding to the X chromosomes by H2A.Z/HTZ-1. PLoS Genet 5: e1000699. doi: 10.1371/journal.pgen.1000699.

Pope LH, Xiong C, Marko JF. 2006. Proteolysis of mitotic chromosomes induces gradual and anisotropic decondensation correlated with a reduction of elastic modulus and structural sensitivity to rarely cutting restriction enzymes. Mol Biol Cell 17: 104-113.

Postow L, Crisona NJ, Peter BJ, Hardy CD, Cozzarelli NR. 2001. Topological challenges to DNA replication: Conformations at the fork. Proc Natl Acad Sci 98: 8219-8226.

Rabitsch KP, Petronczki M, Javerzat JP, Genier S, Chwalla B, Schleiffer A, Tanaka TU, Nasmyth K. 2003. Kinetochore recruitment of two nucleolar proteins is required for homolog segregation in meiosis I. Dev Cell 4: 535-548.

Rawlings JS, Gatzka M, Thomas PG, Ihle JN. 2011. Chromatin condensation via the condensin II complex is required for peripheral T-cell quiescence. EMBO J 30: 263-276.

Renshaw MJ, Ward JJ, Kanemaki M, Natsume K, Nédélec FJ, Tanaka TU. 2010. Condensins promote chromosome recoiling during early anaphase to complete sister chromatid separation. Dev Cell 19: 232-244.

Resnick T, Dej K, Xiang Y, Hawley R, Ahn C, Orr-Weaver T. 2008. Mutations in the chromosomal passenger complex and the condensin complex differentially affect synaptonemal complex disassembly and metaphase I configuration in Drosophila female meiosis. Genetics 181: 875-887.

Ribeiro S, Gatlin J, Dong Y, Joglekar A, Cameron L, Hudson D, Farr C, McEwen B, Salmon E, Earnshaw W, et al. 2009. Condensin regulates the stiffness of vertebrate centromeres. Mol Biol Cell 20: 2371-2380.

Roca J. 2009. Topoisomerase II: A fitted mechanism for the chromatin landscape. Nucleic Acids Res 37: 721-730.

Sakai A, Hizume K, Sutani T, Takeyasu K, Yanagida M. 2003. Condensin but not cohesin SMC heterodimer induces DNA reannealing through protein-protein assembly. EMBO I 22: 2764-2775.

Sakamoto T, Inui YT, Uraguchi S, Yoshizumi T, Matsunaga S, Mastui M, Umeda M, Fukui K, Fujiwara T. 2011. Condensin II alleviates DNA damage and is essential for tolerance of boron overload stress in Arabidopsis. Plant Cell 23: 3533-3546.

Savvidou E, Cobbe N, Steffensen S, Cotterill S, Heck MMS. 2005. Drosophila CAP-D2 is required for condensin complex stability and resolution of sister chromatids. J Cell Sci 118: 2529-2543.

Schleiffer A, Kaitna S, Maurer-Stroh S, Glotzer M, Nasmyth K, Eisenhaber F. 2003. Kleisins: A superfamily of bacterial and eukaryotic SMC protein partners. Mol Cell 11: 571-575.

Schwartz MA, Shapiro L. 2011. An SMC ATPase mutant disrupts chromosome segregation in Caulobacter. Mol Microbiol 82: 1359-1374.

Seipold S, Priller F, Goldsmith P, Harris W, Baier H, AbdelilahSeyfried S. 2009. Non-SMC condensin I complex proteins control chromosome segregation and survival of proliferating cells in the zebrafish neural retina. BMC Dev Biol 9: 40. doi: 10.1186/1471-213X-9-40.

Setoguchi K, Watanabe T, Weikard R, Albrecht E, Kühn C, Kinoshita A, Sugimoto Y, Takasuga A. 2011. The SNP
c.1326T>G in the non-SMC condensin I complex, subunit G (NCAPG) gene encoding a p.Ile442Met variant is associated with an increase in body frame size at puberty in cattle. Anim Genet 42: 650-655.

Shintomi K, Hirano T. 2011. The relative ratio of condensin I to II determines chromosome shapes. Genes Dev 25: 14641469.

Siddiqui NU, Stronghill PE, Dengler RE, Hasenkampf CA, Riggs CD. 2003. Mutations in Arabidopsis condensin genes disrupt embryogenesis, meristem organization and segregation of homologous chromosomes during meiosis. Development 130: 3283-3295.

Siddiqui NU, Rusyniak S, Hasenkampf CA, Riggs CD. 2006. Disruption of the Arabidopsis SMC4 gene, AtCAP-C, compromises gametogenesis and embryogenesis. Planta 223: 990-997.

Smith ED, Xu Y, Tomson BN, Leung CG, Fujikawa Y, Orkin SH, Crispino JD. 2004. More than blood, a novel gene required for mammalian postimplantation development. Mol Cell Biol 24: $1168-1173$.

Stear JH, Roth MB. 2002. Characterization of HCP-6, a C. elegans protein required to prevent chromosome twisting and merotelic attachment. Genes Dev 16: 1498-1508.

Steffensen S, Coelho PA, Cobbe N, Vass S, Costa M, Hassan B, Prokopenko SN, Bellen H, Heck MMS, Sunkel CE. 2001. A role for Drosophila SMC4 in the resolution of sister chromatids in mitosis. Curr Biol 11: 295-307.

Stephens AD, Haase J, Vicci L, Taylor RM, Bloom K. 2011. Cohesin, condensin, and the intramolecular centromere loop together generate the mitotic chromatin spring. I Cell Biol 193: $1167-1180$.

St-Pierre J, Douziech M, Bazile F, Pascariu M, Bonneil E, Sauvé V, Ratsima H, D'Amours D. 2009. Polo kinase regulates mitotic chromosome condensation by hyperactivation of condensin DNA supercoiling activity. Mol Cell 34: 416-426.

Stray JE, Lindsley JE. 2003. Biochemical analysis of the yeast condensin Smc2/4 complex: An ATPase that promotes knotting of circular DNA. J Biol Chem 278: 26238-26248.

Stray JE, Crisona NJ, Belotserkovskii BP, Lindsley JE, Cozzarelli NR. 2005. The Saccharomyces cerevisiae Smc2/4 condensin compacts DNA into $(+)$ chiral structures without net supercoiling. J Biol Chem 280: 34723-34734.

Strick TR, Kawaguchi T, Hirano T. 2004. Real-time detection of single-molecule DNA compaction by condensin I. Curr Biol 14: $874-880$.

Sullivan NL, Marquis KA, Rudner DZ. 2009. Recruitment of SMC by ParB-parS organizes the origin region and promotes efficient chromosome segregation. Cell 137: 697-707.

Sutani T, Yuasa T, Tomonaga T, Dohmae N, Takio K, Yanagida M. 1999. Fission yeast condensin complex: Essential roles of non-SMC subunits for condensation and cdc2 phosphorylation of Cut3/SMC4. Genes Dev 13: 2271-2283.

Swedlow JR, Hirano T. 2003. The making of the mitotic chromosome: Modern insights into classical questions. Mol Cell 11: 557-569.

Tada K, Susumu H, Sakuno T, Watanabe Y. 2011. Condensin association with histone $\mathrm{H} 2 \mathrm{~A}$ shapes mitotic chromosomes. Nature 474: 477-483.

Takemoto A, Kimura K, Yanagisawa J, Yokoyama S, Hanaoka F. 2006. Negative regulation of condensin I by CK2-mediated phosphorylation. EMBO I 25: 5339-5349.

Takemoto A, Murayama A, Katano M, Urano T, Furukawa K, Yokoyama S, Yanagisawa J, Hanaoka F, Kimura K. 2007. Analysis of the role of Aurora B on the chromosomal targeting of condensin I. Nucleic Acids Res 35: 2403-2412. 
Takemoto A, Maeshima K, Ikehara T, Yamaguchi K, Murayama A, Imamura S, Imamoto N, Yokoyama S, Hirano T, Watanabe Y, et al. 2009. The chromosomal association of condensin II is regulated by a noncatalytic function of PP2A. Nat Struct Mol Biol 16: 1302-1308.

Thompson M, Haeusler RA, Good PD, Engelke DR. 2003. Nucleolar clustering of dispersed tRNA genes. Science 302: 1399-1401.

Thornton GK, Woods CG. 2009. Primary microcephaly: Do all roads lead to Rome? Trends Genet 25: 501-510.

Tibelius A, Marhold J, Zentgraf H, Heilig C, Neitzel H, Ducommun B, Rauch A, Ho A, Bartek J, Krämer A. 2009. Microcephalin and pericentrin regulate mitotic entry via centrosome-associated Chk1. J Cell Biol 185: 1149-1157.

Trimborn M, Bell SM, Felix C, Rashid Y, Jafri H, Griffith PD, Neumann LM, Krebs A, Reis A, Sperling K, et al. 2004. Mutations in microcephalin cause aberrant regulation of chromosome condensation. Am J Hum Genet 75: 261266.

Trimborn M, Schindler D, Neitzel H, Hirano T. 2006. Misregulated chromosome condensation in MCPH1 primary microcephaly is mediated by condensin II. Cell Cycle 5: 322-326.

Tsai CJ, Mets DG, Albrecht MR, Nix P, Chan A, Meyer BJ. 2008. Meiotic crossover number and distribution are regulated by a dosage compensation protein that resembles a condensin subunit. Genes Dev 22: 194-211.

Tsang CK, Li H, Zheng XFS. 2007. Nutrient starvation promotes condensin loading to maintain rDNA stability. $E M B O ~ J ~ \mathbf{2 6 :}$ 448-458.

Umbarger MA, Toro E, Wright MA, Porreca GJ, Baù D, Hong S-H, Fero MJ, Zhu LJ, Marti-Renom MA, McAdams HH, et al. 2011. The three-dimensional architecture of a bacterial genome and its alteration by genetic perturbation. Mol Cell 44: 252-264.

Vagnarelli P, Hudson D, Ribeiro SA, Trinkle-Mulcahy L, Lai F, Farr CJ, Lamond AI, Earnshaw WC. 2006. Condensin and Repo-Man-PP1 co-operate in the regulation of chromosome architecture during mitosis. Nat Cell Biol 8: 1133-1142.

Viera A, Gómez R, Parra MT, Schmiesing JA, Yokomori K, Rufas JS, Suja JA. 2007. Condensin I reveals new insights on mouse meiotic chromosome structure and dynamics. PLOS ONE 2: e783. doi: 10.1371/journal.pone.0000783.

Woo J-S, Lim J-H, Shin H-C, Suh M-K, Ku B, Lee K-H, Joo K, Robinson H, Lee J, Park S-Y, et al. 2009. Structural studies of a bacterial condensin complex reveal ATP-dependent disruption of intersubunit interactions. Cell 136: 85-96.

Wood J, Li K, Liang Y, Chen J. 2008. Microcephalin/MCPH1 associates with the condensin ii complex to function in homologous recombination repair. J Biol Chem 283: 2958629592.

Wood AJ, Severson AF, Meyer BJ. 2010. Condensin and cohesin complexity: The expanding repertoire of functions. Nat Rev Genet 11: 391-404.

$\mathrm{Xu}$ Y, Leung CG, Kennedy BK, Crispino JD. 2006. MTB, the murine homologue of condensin II subunit CAP-G2, represses transcription and promotes erythroid cell differentiation. Leukemia 20: 1261-1269.

Yamashita D, Shintomi K, Ono T, Gavvovidis I, Schindler D, Neitzel H, Trimborn M, Hirano T. 2011. MCPH1 regulates chromosome condensation and shaping as a composite modulator of condensin II. J Cell Biol 194: 841-854.

Yanagida M. 2009. Clearing the way for mitosis: Is cohesin a target? Nat Rev Mol Cell Biol 10: 489-496.

Yao M-C, Chao J-L. 2005. RNA-guided DNA deletion in Tetrahymena: An RNAi-based mechanism for programmed genome rearrangements. Annu Rev Genet 39: 537-559.
Yeong FM, Hombauer $\mathrm{H}$, Wendt $\mathrm{KS}$, Hirota $\mathrm{T}$, Mudrak I, Mechtler K, Loregger T, Marchler-Bauer A, Tanaka K, Peters J-M, et al. 2003. Identification of a subunit of a novel kleisin$\beta /$ SMC complex as a potential substrate of protein phosphatase 2A. Curr Biol 13: 2058-2064.

Yu H-G, Koshland DE. 2003. Meiotic condensin is required for proper chromosome compaction, SC assembly, and resolution of recombination-dependent chromosome linkages. J Cell Biol 163: 937-947.

Yu H-G, Koshland D. 2005. Chromosome morphogenesis: Condensin-dependent cohesin removal during meiosis. Cell 123: 397-407. 


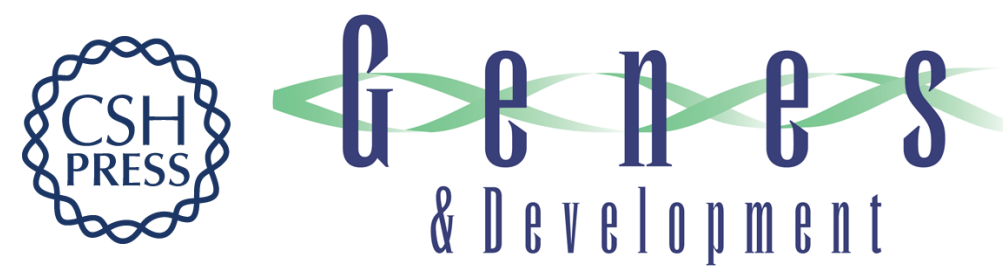

\title{
Condensins: universal organizers of chromosomes with diverse functions
}

\author{
Tatsuya Hirano
}

Genes Dev. 2012, 26:

Access the most recent version at doi:10.1101/gad.194746.112

$\begin{array}{ll}\text { References } & \begin{array}{l}\text { This article cites } 168 \text { articles, } 67 \text { of which can be accessed free at: } \\ \text { http://genesdev.cshlp.org/content/26/15/1659.full.html\#ref-list-1 }\end{array}\end{array}$

License Email Alerting
Service

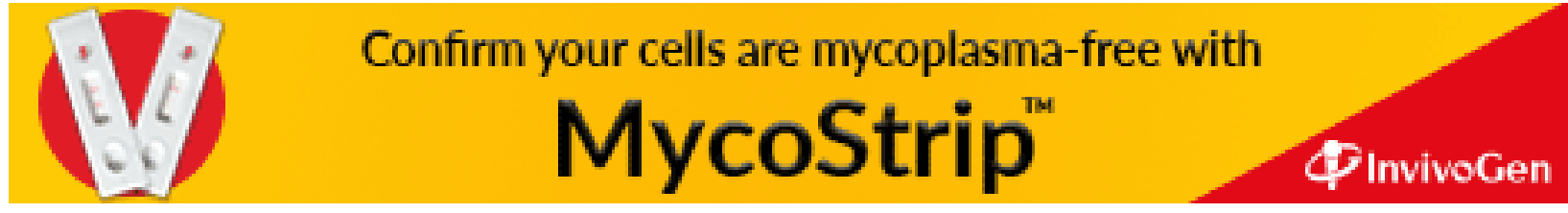

